HIGH FIELD MAGNETORESISTANCE AND DE HAAS-VAN ALPHEN EFFECT

$$
\text { IN ANTIFERromagnetic } \operatorname{PrB}_{6} \text { AND } \mathrm{NdB}_{6}{ }^{*}
$$

Y. Onuki,,$^{* *}$ A. Umezawa, ${ }^{\dagger}$ W. K. Kwok, ${ }^{\dagger \dagger}$ and G. W. Crabtre MAR 201989

Materials Sclence Division

Argonne National Laboratory, Argonne, Illinois 60439

M. Nishihara, T. Yamazaki, T. Omi, and T. Komatsubara

Institute of Materials Science

University of Tsukuba, Sakura-mura, Ibarak1 305, Japan

August 1987

\title{
DISCLAIMER
}

This report was prepared as an account of work sponsored by an agency of the United States Government. Neither the United States Government nor any agency thereof, nor any of their employees, makes any warranty, express or implied, or assumes any legal liability or responsibility for the accuracy, completeness, or usefulness of any information, apparatus, product, or process disclosed, or represents that its use would not infringe privately owned rights. Reference herein to any specific commercial product, process, or service by trade name, trademark, manufacturer, or otherwise does not necessarily constitute or imply its endorsement, recommendation, or favoring by the United States Government or any agency thereof. The views and opinions of authors expressed herein do not necessarily state or reflect those of the United States Government or any agency thereof.

\footnotetext{
*Work supported by the U.S. Department of Energy, BES-Materials Sciences, under Contract \$W-31-109-ENG-38.

** Permanent address: Institute of Material Sclence, Unfversity of Tsukuba, Sakura-mura, Ibarak1 305, Japan.

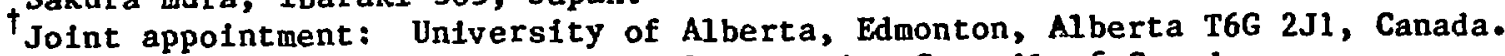
Funded by the Natural Science and Engineering Council of Canada.

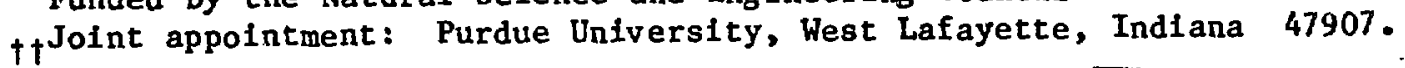




\section{High Field Magnetoresistance and de Haas-van Alphen Effect in Antiferromagnetic $\mathrm{PrB}_{6}$ and $\mathrm{NdB}_{6}$}

Y. Onuki", A. Umezawa" , W. K. Kwok "t* and G. W. Crabtree

Material Scienco Division, Argonno National Laboratony, Argonne, Illinois 60439

M. Nishihara, T. Yamazaki, T. Omi and T. Komatsubara

Institute of Materials Science, University of Tsukuba, Sakura - mura, Baraki 305, Japan

- Permanent Address: Institute of Material Science, University of

Tsukuba, Sakura-mura, Ibaraki 305, Japan

* Joint appointment: University of Alberta, Edmonion, Alberta T6G 2J1, Canada. Funded by the Natural Science and Engineering Council of Canada

** Joint appointment: Purdue University, W. Lafayette, Indiana 47907 


\begin{abstract}
The transport properties and the de Haas-van Alphen (dHvA) effect have been measured for antiferromagnetic $\mathrm{PrB}_{6}$ and $\mathrm{NdB}_{6}$. The number of conduction electrons is approximately one per unit cell. The magnetoresistance shows the existence of open orbits implying a multiply connected Fermi surface. The angular dependence of the magnetoresistance is roughly similar to that of the reference material, $\mathrm{LaB}_{6}$. The dHvA data in $\mathrm{PrB}_{6}$ shows both paramagnetic and antiferromagnetic Fermi surfaces. The antiferromagnetic Fermi surface arises from new magnetic Brillowin zone boundaries and antiferromagnetic gaps introduced by the magnetic order, and the paramagnetic Fermi surface from magnetic breakdown through the small antiferromagnetic gaps in high field. Hybridization between the conduction electrons and the felectrons has been observed through the cyclotron masses, which in $\mathrm{PrB}_{6}$ are three times larger than the corresponding masses of $\mathrm{LaB}_{6}$. In $\mathrm{NdB}_{6}$ only the antiferromagnetic Fermi surface, quite different from those of $\mathrm{LaB}_{6}$ and $\mathrm{PrB}_{6}$, has been observed.
\end{abstract}




\section{INTRODUCTION}

In many rare earth and actinide compounds the f electrons show a wide variety of behavior including Pauli itinerance, spin fluctuations, mixed valence and heavy fermion, and local moment magnetic order ${ }^{1-3}$. The rare earth hexaborides $\mathrm{RB}_{6}$ are an interesting series of compounds where systematic felectron behavior including many of these features can be studied. $\mathrm{La}_{\mathrm{a}} \mathrm{B}_{6}$ is a non-f electron transition . metal-like system, $\mathrm{CeB}_{6}$ is a typical Kondo substance, $\mathrm{PrB}_{6}$ and $\mathrm{NdB}_{6}$ are local moment metals and $\mathrm{SmB}_{6}$ is a mixed valence compound with a gap in the narrow $4 \mathrm{f}$ band just at the Fermi level. Many experimental and theoretical studies are focussed on the peculiar $4 \mathrm{f}$ behavior of these substances.

If the $4 \mathrm{f}$ electrons are completely localized, $\mathrm{R}^{3+}$, there is no hybridization between the $4 \mathrm{f}$ electrons and the conduction electrons. The Fermi surface and cyclotron mass are almost the same as those of the non-f La compounds. In this case the $4 f$ electrons act only as scattering centers for the conduction electrons. However, as the $4 f$ states approach the Fermi level in energy in going from $\mathrm{Gd}$ to $\mathrm{Ce}$, the $\mathrm{f}$ electrons hybridize more strongly with the conduction electrons. The most highly hybridized state is expected in the $\mathrm{Ce}$ compound. In $\mathrm{RB}_{6}$ the Ce compound displays a large electronic specific heat typical of $f$ electron hybridization, yet still retains a large enough moment to order magnetically at low temperature ${ }^{4-6}$. The reduced moment and logarithmic like resistivity in $\mathrm{CeB}_{6}$ are typical of the Kondo effect.

In general, the RKKY interaction competes with the Kondo effect by creating an exchange coupling between $f$ moments which leads to magnetic order at low temperatures. As the number of $f$ electrons increases in the $\mathrm{RB}_{6}$ series the moment becomes larger and the $\mathrm{RKKY}$ interaction stronger, leading to magnetic order. temperatures which roughly follow the de Gennes relation. The antiferromagnetic ordering temperatures in $\mathrm{CeB}_{6}, \mathrm{PrB}_{6}$, and $\mathrm{NdB}_{6}$ are $2.4 \mathrm{~K}, 7.0 \mathrm{~K}$, and $7.8 \mathrm{~K}$, respectively $4-7$, roughly following the de Gennes relation.

The presence of the $f$ electron in $\mathrm{RB}_{6}$ alters the Fermi surface in three ways: through hybridization with the conduction electrons, through the $f$ electron contribution to the crystal potential, and through the introduction of new magnetic Brillouin zone boundaries and magnetic energy gaps which occur when the f electron moments order. 
The first two mechanisms alter the electronic band structure of the metal and may affect both the Fermi surface geometry and cyclotron effective masses. The two mechanisms are difficult to separate from each other, although some separation can be achieved ${ }^{8}$ by careful Fermi surface measurements and band structure calculations. The effect of the third mechanism can be approximated by a band folding procedure where the paramagnetic Fermi surface containing all the corrections due to hybridization and crystal potential effects is folded into the smaller Brillouin zone induced by the antiferromagnetic order. If the magnetic energy gaps associated with the antiferromagnetic structure are small enough, electrons undergoing cyclotron motion in the presence of a magnetic field can tunnel through these gaps and follow orbits on the paramagnetic Fermi surface. If this magnetic breakdown occurs, the paramagnetic Fermi surface may be observed in the de Haas van Alphen ( $\mathrm{dHvA}$ ) effect even in the presence of magnetic order. However, if there is significant hybridization or a large contribution of the $f$ electron to the crystal potential, the paramagnetic Fermi surface geometry may be somewhat different from the non-f analog metal. Generally, one expects hybridization to appear in the dHvA results as an increased cyclotron effective mass due to the admixture of the heavier $f$ electron with the spd conduction electrons. Such effects have been observed ${ }^{9-11}$ in $\mathrm{CeSn}_{3}, \mathrm{CeCu}_{6}$, and $\mathrm{UPt}_{3}$.

The first investigations of the Fermi surfaces of the $\mathrm{RB}_{6}$ compounds were done on $\mathrm{LaB}_{6}$ by Arko, et al. 12,13 using magnetoresistance and the de Haas-van Alphen (dHvA) effect in fields . up to $13.2 \mathrm{~T}$ and temperatures down to $0.35 \mathrm{~K}$ and by Ishizawa, et al. 14,15 using the dHvA effect in fields up to $6 \mathrm{~T}$ and at temperatures as low as $1.5 \mathrm{~K}$. The experimental results were interpreted by a Fermi surface model consisting of large electron sheets at the $X$ point of the Brillouin zone connected by tiny necks in the $\mathrm{X}-\mathrm{X}$ direction as illustrated in Figure 1. Band structure calculations carried out by Walch, et al. 16 and by Hasegawa and Yanase 17 confirmed the general topology of the empirical Fermi surface model but were unable to provide a quantitative explanation of all the observed orbits. However, the geometry of the electron sheets at $X$ which contain nearly all the electrons and determine the electronic behavior were qualitatively well explained.

Systematic Fermi surface measurements on $\mathrm{LaB}_{6}, \mathrm{CeB}_{6}, \mathrm{PrB}_{6}$, and 
$\mathrm{NdB}_{6}$ were first carried out by van Deursen, et al. ${ }^{18}$ for the single field direction $\langle 100\rangle$ using pulsed fields up to $33 \mathrm{~T}$ at temperatures down to 1.3K. They observed one frequency in $\mathrm{CeB}_{6}$ and up to five frequencies in the other compounds which suggested the Fermi surface topology for all the compounds was similar. Later work ${ }^{19}$ on the anisotropy of the frequencies in $\mathrm{CeB}_{6}$ and $\mathrm{PrB}_{6}$ confirmed the existence of the $\mathrm{X}$-centered ellipsoids in $\mathrm{PrB}_{6}$ and revealed some additional features not directly analogous to $\mathrm{LaB}_{6}$ which suggested the existence of smaller closed sheets.

An independent study of the Fermi surface of $\mathrm{PrB}_{6}$ using the dHvA effect and magnetoresistance in fields of $15 \mathrm{~T}$ at temperatures down to $1.4 \mathrm{~K}$ by Onuki, et al. ${ }^{20}$ provided a more complete picture of the Fermi surface geometry and effective masses in $\mathrm{PrB}_{6}$. This work revealed two sets of low frequency orbits with the same anisotropy as those arising from the $\mathrm{X}-\mathrm{X}$ neck orbits in $\mathrm{LaB}_{6}$ but with areas approximately 100 times and 30 times larger than those in $\mathrm{LaB}_{6}$. This more extensive data generally agreed with those of van Deursen, et al, for the areas and masses where comparison was possible.

... In this paper we continue investigations of the Fermi surface properties of the $\mathrm{RB}_{6}$ series with magnetoresistance and Hall effect measurements in $\mathrm{CeB}_{6}, \mathrm{PrB}_{6}$, and $\mathrm{NdB}_{6}$ and with additional $\mathrm{dHvA}$ measurements in $\mathrm{NdB}_{6}$ in fields up to $15 \mathrm{~T}$ and temperatures down to $0.4 \mathrm{~K}$. In $\mathrm{CeB}_{6}$ we find features characteristic of the Kondo effect. In $\mathrm{PrB}_{6}$ we present new data on the open orbits from the magnetoresistance. In $\mathrm{NdB}_{6}$ we present for the first time the detailed variation of the dHvA frequencies and effective masses with magnetic field direction. We compare our data for all three rare earth compounds with those of $\mathrm{LaB}_{6}$ and discuss the implications for models of the $f$ electron behavior.

\section{EXPERIMENTAL PROCEDURES}

Single crystals of $\mathrm{RB}_{6}$ were grown by a floating zone technique. Three passes of the molten zone were made on each crystal in an atmosphere of $\mathrm{Ar}$ at 30 bar to prevent excessive evaporation. The typical dimensions of the resulting crystalline bars were $8 \mathrm{~mm}$ in diameter and $40 \mathrm{~mm}$ in length. 
The electrical resistivity, magnetoresistance and Hall coefficient were measured by a DC method. The Hall coefficient was measured in the temperature range of $0.7 \mathrm{~K}$ to room temperature in a field of $0.8 \mathrm{~T}$, where the Hall voltage was linear in the field. The magnetoresistance was measured at $1.4 \mathrm{~K}$ in fields up to $15 \mathrm{~T}$. The $\mathrm{dHvA}$ experiments were carried out at $0.4 \mathrm{~K}$ in fields up to $15 \mathrm{~T}$ using field modulation and second harmonic detection. The dHvA frequencies were obtained by taking fast Fourier transforms of the oscillatory magnetization signal. Effective masses were determined from the temperature dependence of the amplitude of the signal at fixed field.

\section{EXPERIMENTAL RESULTS AND ANALYSIS}

The temperature dependence of the electrical resistivity of single crystal samples of $\mathrm{RB}_{6}$ for the current along the $\langle 100\rangle$ direction is shown in Fig. 2. The residual resistivity ratio $\rho_{R T} / \rho_{1.4 K}$ is 220 in $\mathrm{PrB}_{6}$ and 120 in $\mathrm{NdB}_{6}$. The inset shows the steep decrease of the resistivity below $7.0 \mathrm{~K}$ in $\mathrm{PrB}_{6}$ and $7.8 \mathrm{~K}$ in $\mathrm{NdB}_{6}$ which is due to antiferromagnetic ordering in these compounds 6,7 . Two steps at $7.0 \mathrm{~K}$ and $4.2 \mathrm{~K}$ in $\mathrm{PrB}_{6}$ correspond to the appearance of incommensurate and commensurate spin structures, respectively. The resistivity of $\mathrm{CeB}_{6}$ shows a dense Kondo behavior, increasing logarithmically with decreasing temperature until it passes through a maximum at $4.0 \mathrm{~K}$ and decreases quite rapidly at lower temperatures. $\mathrm{CeB}_{6}$ undergoes two ordering transitions at $3.2 \mathrm{~K}$ and $2.3 \mathrm{~K}$, where the lower one is due to the onset of antiferromagnetic order. $4,5,21,22$

The temperature dependence of the Hall coefficient in single crystal $\mathbf{R B}_{\mathbf{6}}$ is shown in Fig.3. The Hall coefficient is almost temperature independent, except for the region near the ordering temperature of $\mathrm{CeB}_{6}$. The present results are $-(4.5 \pm 0.7) \times 10^{-4} \mathrm{~cm}^{3} /$ coul. in $\mathrm{CeB}_{6}$, $-(3.9 \pm 1.1) \times 10^{-4} \mathrm{~cm}^{3} /$ coul. in $\mathrm{PrB}_{6}$ and $-(4.6 \pm 0.9) \times 10^{-4}$ $\mathrm{cm}^{3} /$ coul. in $\mathrm{NdB}_{6}$. The Hall coefficient of $\mathrm{LaB}_{6}$ was measured by Tanaka et al. 23 to be $-4.5 \times 10^{-4} \mathrm{~cm}^{3} /$ coul. Using the lattice constants of $\mathrm{RB}_{6}$ (R:La ,4.156 $\AA$; Ce, 4.140 $\AA$; $\operatorname{Pr}, 4.133 \AA$ and Nd, 4.126 $\AA$ ) we calculate the number of conduction electron per unit cell to be 1.0 , $1.0 \pm 0.1,1.2 \pm 0.3$, and $1.0 \pm 0.2$, respectively. These values of the electron densities are consistent with the Fermi surface of $\mathrm{LaB}_{6}$, which fills half the Brillouin zone with the three nearly spherical ellipsoids at 
$X$. Band calculations of $\mathrm{LaB}_{6}$ show that the electron states at the Fermi surface are mainly of 5d-character implying that they originate from the rare earth atom.

We show in Fig.4 the angular dependence of the transverse magnetoresistance $\Delta \rho / \rho=\{\rho(H)-\rho(0)\} / \rho(0)$ of $R_{6}$ in the $\{100\}$ plane, and in Fig.5 the field dependence of the transverse magnetoresistance in selected field directions. The data of $\mathrm{LaB}_{6}$ has been taken from ref.24. When the field is applied along the $\langle 100\rangle$ direction, the magnetoresistance saturates at high fields in $\mathrm{LaB}_{6}, \mathrm{PrB}_{6}$ and $\mathrm{NdB}_{6}$. Non-saturating behavior is observed in all other directions in the $\{100\}$ plane. For uncompensated metals like $\mathrm{LaB}_{6}$, this behavior is characteristic of the existence of an open orbit along the $<100\rangle$ current direction.

We have observed a complicated behavior in the magnetoresistance of $\mathrm{PrB}_{6}$. The magnetoresistance saturates above $100 \mathrm{kOe}$ for fields directions between about $5^{\circ}$ to $40^{\circ}$, and $55^{\circ}$ to $85^{\circ}$ from $\langle 100\rangle$. This behavior is due to magnetic breakdown effects resulting in topological changes of the orbit and is not found in $\mathrm{NdB}_{6}$. The oscillatory magnetoresistance in $\mathrm{LaB}_{6}$ is due to Shubnikov - de Haas oscillations on the small neck connecting the $X$-centered ellipsoids. We note that both. $\mathrm{PrB}_{6}$ and $\mathrm{NdB}_{6}$ are in the antiferromagnetic phase for the fields and temperatures employed in the magnetoresistance experiments. Our preliminary magnetization measurements show that this antiferromagnetic phase is stable up to at least $400 \mathrm{kOe}$. We also stress that the magnitude of $\Delta \rho / p \sim 20$ at $150 \mathrm{kOe}$ in the open orbit direction is consistent with the high field condition, $\omega_{c} \tau>1$ because the relationship $\Delta \rho / \rho=\left(\omega_{c} \tau\right)^{2}$ approximately holds in the non-saturating magnetoresistance. Here, $\omega_{c}$ is the cyclotron frequency and $\tau$ is the reiaxation time of the carrier. The value of $\omega_{c} \tau$ is the number of the cyclotron orbits completed between scattering events.

The magnetoresistance of $\mathrm{CeB}_{6}$ is negative, which is characteristic to the Kondo effect. In the low field region the negative magnetoresistance is related to magnetization $M$ by $\Delta \rho / \rho \propto-M^{2}$. At high fields above $100 \mathrm{kOe}$, the magnetoresistance in the field direction of $23.5^{\circ}$ increases slightly with field. We suppose that the positive magnetoresistance due to the cyclotron motion of the carriers is added to the negative magnetoresistance mentioned above. The magnetoresistance is known to become positive 25 at $0.5 \mathrm{~K}$ above 50 
kOe. Apparently at high enough field and low enough temperature, the magnetization dependent negative magnetoresistance saturates, leaving the positive magnetoresistance due to Fermi surface effects. In this low temperature, high field region topological information on the Fermi surface of $\mathrm{CeB}_{6}$ could be determined.

The angular dependence and field dependence of the transverse magnetoresistance in the $\{110\}$ plane are shown in Figs 6 and 7, respectively. Non-saturating behavior due to the existence of open orbits is found in the region of $1^{\circ}-10^{\circ}$ and $20^{\circ}-35^{\circ}$ from $\langle 100\rangle$ in $\mathrm{LaB}_{6}$. Similar behavior is observed in $\mathrm{NdB}_{6}$ in the region of $1^{\circ}-24^{\circ}$ and $42^{\circ}-55^{\circ}$ from $<100>. \operatorname{PrB}_{6}$ does not show such non-saturating behavior in the vicinity of the $<100\rangle$ direction. The non-saturating behavior is only found in the region of $55^{\circ}$ to $77^{\circ}$ from $\left.<100\right\rangle$. Although the $\langle 100\rangle$ direction is the so-called singular field direction in $\mathrm{LaB}_{6}$ and $\mathrm{NdB}_{6}$, it is not so in $\mathrm{PrB}_{6}$.

We summarize the open orbit directions derived from the magnetoresistance data in Table 1. The existence of open orbits confirms the multiply connected nature of the Fermi surface and can be understood qualitatively on the basis of the Fermi surface model of Fig. 1 as explained further below.

Finally, we show in Fig. 8 the angular dependence of $\mathrm{dHvA}$ frequencies in $\mathrm{RB}_{6}$. The data of $\mathrm{LaB}_{6}$ has been taken from refs. 14 and 15. The $<100\rangle$ frequencies and the corresponding cyclotron masses which have been determined from the temperature dependence of the dHvA oscillations are tabulated in Table 2.

Although there is a great deal of detailed data concerning the Fermi surfaces of the $\mathrm{RB}_{6}$ compounds in Fig.8, there are some systematic features which can be qualitatively understood with a simple picture. We note that there are many similarities in the $\mathrm{dHvA}$ data of $\mathrm{LaB}_{6}$ and $\mathrm{PrB}_{6}$, but almost no similarity between the data in $\mathrm{LaB}_{6}$ and those in $\mathrm{NdB}_{6}$. Close examination reveals that the $\alpha, \gamma, \varepsilon, \delta, \lambda$, and $\xi$ branches in $\mathrm{LaB}_{6}$ correspond in angular dependence and magnitude approximately with branches in $\mathrm{PrB}_{6}$. These branches have been so labeled in the data for $\mathrm{PrB}_{6}$ in Fig.8. The other branches in $\mathrm{PrB}_{6}$ which do not correspond to branches in $\mathrm{LaB}_{6}$ have been labeled with arabic letters. The $\alpha$ frequency in $\operatorname{PrB}_{6}$ has three branches $\alpha_{1}, \alpha_{2}$, and $\alpha_{3}$ in the $\{100\}$ plane, and it degenerates into two in the $\{110\}$ plane, as is observed in $\mathrm{LaB}_{6}$. The cyclotron mass of the $\alpha_{3}$ orbit in 
$\operatorname{PrB}_{6}, 1.95 \mathrm{~m}_{0}$, is three times larger than the corresponding mass in $\mathrm{LaB}_{6}, 0.61-0.64 \mathrm{~m}_{\mathrm{o}}$. Except for the $\rho$ and $\xi$ oscillations, all of the long range frequencies in $\mathrm{LaB}_{6}$ are also observed in $\mathrm{PrB}_{6}$.

The $g$ and $g^{\prime}$ branches in $\mathrm{PrB}_{6}$ are very similar in shape to the $\rho$ branches in $\mathrm{LaB}_{6}$ but are much larger in magnitude. The dHvA frequencies of $\mathrm{g}$ and $\mathrm{g}^{\prime}$ in the $<100>$ direction are $5.9 \times 10^{6} \mathrm{Oe}$ and 1.5 $x 10^{6} \mathrm{Oe}$, respectively, which are one hundred times and thirty times larger than $5.2 \times 10^{4} \mathrm{Oe}$ as observed in $\mathrm{LaB}_{6}$. The masses on these orbits are $0.62 \mathrm{~m}_{\mathrm{o}}$ and $0.28 \mathrm{~m}_{\mathrm{o}}$, heavier than the corresponding value $0.046 \mathrm{~m}_{0}$ in $\mathrm{LaB}_{6}$. In $\mathrm{LaB}_{6}$ these branches arise from the necks which connect the X-centered ellipsoids. We propose that a similar feature exists in $\mathrm{PrB}_{6}$, except that there are two necks connecting each ellipsoid. and the necks are appropriately larger. 20

The similarity of the Fermi surfaces of $\mathrm{LaB}_{6}$ and $\mathrm{PrB}_{6}$ is surprising because $\mathrm{PrB}_{6}$ orders with a complicated magnetic structure involving 32 unit cells. ${ }^{6}$ This introduces a magnetic Brillouin zone which is much smaller than the X-centered ellipsoids. When the Fermi surface is folded into the new Brillouin zone, the large orbits such as $\alpha$ are interrupt $\mathrm{l}$ by zone boundaries and should not appear in the data. We explain the similarity of the $\mathrm{dHvA}_{\text {data }}$ in $\mathrm{LaB}_{6}$ and $\mathrm{PrB}_{6}$ by magnetic breakdown, assuming the applied field is strong enough to drive the electrons through the energy gaps created at the magnetic Brillouin zone boundaries by the antiferromagnetic structure. In that case the paramagnetic Fermi surface would be observed, and it may be similar to that of $\mathrm{LaB}_{6}$ if the effects of hybridization and the felectron crystal potential are not too large.

The branches in $\mathrm{PrB}_{6}$ that are not observed in $\mathrm{LaB}_{6}$ we attribute to the antiferromagnetic Fermi surface. These branches represent the paramagnetic Fermi surface fclded into the smaller magnetic Brillouin zone. With incomplete magnetic breakdown both the paramagnetic and antiferromagnetic Fermi surfaces can be seen. The a branch is the lingest frequency on the antiferromagnetic Fermi surface. This signal disappears in the field range of $13^{\circ}$ to $35^{\circ}$ from $\langle 100\rangle$ in the $\{100\}$ plane and $22^{\circ}$ to $77^{\circ}$ from $\langle 100\rangle$ in the $\{110\}$ plane. The Fermi surface sheet giving rise to the a orbit is nearly sphezical, being slightly stretched in the $\langle 110\rangle$ direction and may possess arms which cause the orbit to disappear. These arms are probably in the $\langle 111\rangle$ direction.

Besides the $a, g$ and $g^{\prime}$ branches, three branches $b, b^{\prime}$ and $b^{\prime \prime}$ are 
observed, centered at the $<111\rangle$ direction. van Deursen et al. 19 observed another similar branch with a frequency of $3.7 \times 10^{7} \mathrm{Oe}$ in the $<111>$ direction.

The dHvA frequencies observed in $\mathrm{NdB}_{6}$ are extremely different from those of $\mathrm{LaB}_{6}$ and $\mathrm{PrB}_{6}$. In fact, there are no oscillations which correspond to those of $\mathrm{LaB}_{6}$. The characteristic features in the Fermi surface of $\mathrm{NdB}_{6}$ are as follows. The a branch is a nearly spherical Fermi surface which is slightly stretched in the $\langle 100\rangle$ direction. The signal of the a branch, however, disappears in the field angle of $40^{\circ}$ to $90^{\circ}$ from $\langle 100\rangle$ in the $\{110\}$ plane. Moreover, it becomes extremely weak in the vicinity of the $<100\rangle$ direction, possessing a maximum amplitude at about $10^{\circ}$.

The $g$ and $h$ branches are nearly spherical ellipsoids. As there is no third branch in the $\{100\}$ plane, $g$ and $h$ may not be symmetry equivalent ellipsoids. The signal of the $h$ branch seems to be degenerate with the $g$ signal in the field range of $55^{\circ}$ to $90^{\circ}$. We do not believe that the $\mathrm{h}$ signal simply disappears for angles greater than $55^{\circ}$, because its amplitude is quite strong for directions near $\langle 111\rangle$.

The other branches are limited to small angle regions. The $c$ branch possesses a symmetry at the $\langle 110\rangle$ direction, the $d$ and $j$ branches at the $<110\rangle$ direction, and the $\mathrm{f}$ and $\mathrm{k}$ branches at the $<111\rangle$ direction.

\section{DISCUSSION}

The data presented above can be understood using simple ideas for the effect of the felectron on the Fermi surface through the crystal potential, hybridization, and magnetic order. We argue that the main features of the paramagnetic Femi surfaces of $\mathrm{LaB}_{6}, \mathrm{PrB}_{6}$, and $\mathrm{NdB}_{6}$ are all similar, with large ellipsoids at $X$ connected by necks in the $X-X$ directions. Slight differences in the band structures of the three metals due to the contribution of the $f$ electron to the crystal potential or to direct hybridization of the conduction states with the $f$ electron alter details of the Fermi surface geometry like the shape of the ellipsoids and the size and connectivity of the necks. If significant $f$ electron hybridization occurs, the effective masses of the paramagnetic Fermi surface are increased relative to $\mathrm{LaB}_{6}$. When magnetic order occurs, the paramagnetic Fermi surface must be folded into the smaller 
magnetic Brillouin zone, perhaps altering the observable cyclotron orbits drastically. However, if the magnetic energy gaps at the new zone boundaries are not too large, the paramagnetic Fermi surface can still be seen due to magnetic breakdown. If the applied field range spans the breakdown field, the paramagnetic and antiferromagnetic Fermi surfaces will be seen simultaneously. This case applies to $\operatorname{PrB}_{6}$. If the magnetic energy gaps are too large, the breakdown field will be large compared to the applied field and no magnetic breakdown wili occur. In this case only the antiferromagnetic Fermi surface will be seen, as occurs in $\mathrm{NdB}_{6}$.

First we justify the claim that the main features of the paramagnetic Fermi surfaces of $\mathrm{LaB}_{6}, \mathrm{PrB}_{6}$, and $\mathrm{NdB}_{6}$ are essentially the same. There are three reasons for this assertion. First, the value of the Hall coefficient in $\mathrm{RB}_{6}$ is almost the same at room temperature in all three compounds. The number of conduction electrons is approximately one per unit cell, suggesting that all three metals have the ellipsoidal $\mathrm{X}$-centered surfaces of $\mathrm{LaB}_{6}$. Even below the Néel temperature, the carrier concentration does not change in $\mathrm{PrB}_{6}$ and $\mathrm{NdB}_{6}$ as indicated by the temperature independent of the Hall coefficient. This temperature independence of the Hall coefficient below the Néel temperature is unusual. Secondly, the Fermi surface determined by two-dimensional angular correlation of positron annihilation radiation shows nearly the same shape for all $\mathrm{RB}_{6}$ compounds at room temperature 26 . The positron annihilation results show directly the existence of an electron ellipsoid at the $X$ point. This work confirms the interpretation of the Fermi surface discussed above. Finally, we observe the $\alpha$ orbits arising from the X-centered ellipsoids in both $\mathrm{LaB}_{6}$ and $\mathrm{PrB}_{6}$, but not in $\mathrm{NdB}_{6}$. However, as shown in Table 2, van Deursen et al. ${ }^{18}$ observed four dHvA signals in the $\langle 100\rangle$ field direction in $\mathrm{NdB}_{6}$. Three of them corresponds to the $c, g$ and $h$ branches in the present experiment. The other dHvA frequency is 7.98 $x 10^{7} \mathrm{Oe}$, which corresponds to the $\alpha_{3}$ branch of the X-centered ellipsoids. Their observation of this magnetic breakdown orbit in $\mathrm{NdB}_{6}$ is due to their use of high fields of 29T compared to our highest field of $15 \mathrm{~T}$. Thus the basic feature of the $\mathrm{LaB}_{6}$ Fermi surface is seen by dHvA measurements in both $\mathrm{PrB}_{6}$ and $\mathrm{NdB}_{6}$. Based on the Hall coefficient data, the positron annihilation data, and the high field dHvA data, we conclude that the paramagnetic Fermi surfaces of all three 
metals are very similar.

Despite the basic similarity in the main features of the paramagnetic Fermi surfaces of $\mathrm{LaB}_{6}, \mathrm{PrB}_{6}$, and $\mathrm{NdB}_{6}$ there are small differences in the details of the geometry related to the size and connectivity of the necks. These differences can be seen in the open orbit behavior derived from the magnetoresistance data and in the dHvA data.

First we discuss the magnetoresistance. The magnetoresistance of $\mathrm{NdB}_{6}$ in fields un to $15 \mathrm{~T}$ is very similar to that of $\mathrm{LaB}_{6}$. The analogous features are as follows:

1) The $\langle 100\rangle$ direction is the singular field direction.

2) The open orbit in the $\{100\}$ plane is found in all field directions except the $<100>$ direction.

3) Open orbits of $\mathrm{NdB}_{6}$ in the $\{110\}$ plane exist in the range of $1^{\circ}$ to $24^{\circ}$ and $42^{\circ}$ to $55^{\circ}$, while the one of $\mathrm{LaB}_{6}$ exists in the range of $1^{\circ}$ 10 and $20^{\circ}$ to $35^{\circ}$.

In $\mathrm{PrB}_{6}$, open orbits are observed in the whole region of the $[100\}$ plane except the $<100>$ direction and its vicinity. Unlike in $\mathrm{LaB}_{6}$ and $\mathrm{NdB}_{6},<100>$ is not a singular direction because there is a finite range of angles near $<100>$ in which no open orbit is found. In the $\{110\}$ plane open orbits are found in only one angular range, $55^{\circ}$ to $77^{\circ}$ from $<100>$, instead of two ranges as in $\mathrm{LaB}_{6}$ and $\mathrm{NdB}_{6}$.

The angles at which the open orbits appear and disappear provide information about the placement and size of the necks in the paramagnetic Fermi surface. The open orbits on the $\mathrm{LaB}_{6}$ Fermi surface for various field directions are shown in Fig.9. For the field near the $<100\rangle$ direction in the $(110\}$ plane (Fig.9b) the open orbit trajectory runs between two necks in the region labelled "A". The angular region of the open orbit is restricted by the existence of these two necks.If the Fermi surface of $\mathrm{PrB}_{6}$ is similar to that of $\mathrm{LaB}_{6}$, we infer that the two necks mentioned above are so large that they approximately touch each other, explaining the absence of the open orbit near the $<100>$ direction in the $(110\}$ plane in $\mathrm{PrB}_{6}$. The trajectory of the open orbit shown in Fig. 9 (c) is also restricted by two necks, namely one neck which the orbit must avoid near the $\mathrm{B}$ point and a second neck which is part of the orbit at the $C$ point (the $C$ neck is hidden by an ellipscid in the view of Fig 9a). If the neck is small, this open orbit will be found at angles close to $<100>$. In $\mathrm{LaB}_{6}$, the open orbit first appears at $20^{\circ}$ from $<100>$ in the $[110\}$ plane. As the field 
angle becomes larger than $20^{\circ}$, the path gradually deviates from the C neck eventually forming a closed orbit at approximately $35^{\circ}$. In $\operatorname{PrB}_{6}$, the neck is expected to be relatively large, as confirmed by the appearance of the open orbit at the rather high angle of $55^{\circ}$ from $<100\rangle$ in the $\{110\}$ plane.

...-This larger neck in $\operatorname{PrB}_{6}$ is roughly consistent with the $\mathrm{dHvA}$ results. The $\alpha$ oscillation of $\operatorname{PrB}_{6}$ dișappears over a wider field range than in $\mathrm{LaB}_{6}$, as shown in Table 3, suggesting a larger neck dimension. As discussed in an earlier paper, such a result favors two interconnecting necks whose dimensions are roughly consistent with the $\mathrm{g}$ and $\mathrm{g}^{\prime}$ orbits ${ }^{20}$. However, the length of the necks is very short if the ellipsoid dimensions of $\mathrm{PrB}_{6}$ are the same as those of $\mathrm{LaB}_{6}$. In this case we could not observe the $g$ and $g^{\prime}$ oscillations over a wide field range or, in particular, for the field along the $\langle 100\rangle$ direction.

Therefore if the $\mathrm{g}$ and $\mathrm{g}$ ' branches correspond to the necks in the paramagnetic Fermi surface, it is necessary to modify the shape of the ellipsoids in $\mathrm{PrB}_{6}$.

If the above analysis is also applied to $\mathrm{NdB}_{6}$, the neck of $\mathrm{NdB}_{6}$ is a little larger than that of $\mathrm{LaB}_{6}$ because the open orbit appears at $42^{\circ}$ in the $\{110\}$ plane. This cannot be confirmed with the dHvA results, because only the antiferromagnetic Fermi surface is observed.

The magnetic breakdown phenomena that we invoke to explain the observation of the paramagnetic Fermi surface in $\mathrm{PrB}_{6}$ can be observed on the paramagnetic Fermi surface itself. For example, for the field in the $<100>$ direction the closed $\alpha_{1}$ orbit in $\mathrm{PrB}_{6}$ and $\mathrm{LaB}_{6}$ should not exist because it is interrupted by a neck. However, as shown in Table 2, at sufficiently high fields the $\alpha_{1}$ orbit can be seen, implying magnetic breakdown across the neck, a feature that has been noted in earlier $\mathrm{dHvA}$ and band structure studies of $\mathrm{LaB}_{6}$. In the antiferromagnetic state the degree of magnetic breakdown through the magnetic zone boundary gaps depends on the size of the gaps. We expect these gaps to scale roughly with the magnitude of the magnetic moment or the de Gennes factor. Apparently the magnetic fields used in our experiment are large enough to cause magnetic breakdown through the gaps in $\mathrm{PrB}_{6}$ but are too weak to cause breakdown in $\mathrm{NdB}_{6}$.

Hybridization of the $f$ electron with the conduction electrons can be seen most directly in the cyclotron effective masses. Because the $f$ 
electrons are considerably more localized than the conduction electrons, hybridization with felectrons usually lowers the Fermi velocities and raises the effective masses of the hybridized state. Thus by comparing the effective masses of analogous orbits in $\mathrm{RB}_{6}$, one infers the relative importance of hybridization in the metals. On the paramagnetic Fermi surface of $\mathrm{PrB}_{6}$ the mass of the $\alpha_{3}$ orbit is 1.95 $\mathrm{m}_{0}$, about three times larger than the mass of the corresponding orbit in $\mathrm{LaB}_{6}$. This suggests that the felectron in $\mathrm{PrB}_{6}$ is slightly hybridized with the conduction electrons. The increased mass in $\mathrm{PrB}_{6}$ over $\mathrm{LaB}_{6}$ is a general feature of the paramagnetic Fermi surfaces in the two materials as shown in Fig. 10, where the mass versus frequency is plotted on a logarithmic scale. As shown, there is approximately a factor of three enhancement in all the measured masses in the two materials. If this enhancement is due to a narrowing of the bandwidth from $f$ electron hybridization, then Fig. 10 implies that the hybridization is approximately equally strong for all the conduction electrons in $\mathrm{PrB}_{6}$. This general enhancement of all the masses when $f$ electron hybridization occurs has also been $\operatorname{seen}^{9}$ in $\mathrm{CeSn}_{3}$. The paramagnetic state of $\mathrm{NdB}_{6}$, as measured by van Deursen et al., shows a return to the masses observed in $\mathrm{LaB}_{6}$, suggesting that $f$ hybridization is absent in this metal. This interpretation is consistent with the much larger binding energy of the $f$ electron in $\mathrm{Nd}$ compared to $\mathrm{Pr}$.

In the antiferromagnetic state, the Fermi surface changes due to the appearance of the magnetic zone boundaries can be approximated by folding the paramagnetic Fermi surface into the smaller magnetic Brillouin zone. The magnetic structure in $\mathrm{RB}_{6}$ may be quite complicated even though its chemical structure is simple cubic. The magnetic unit cell of $\mathrm{PrB}_{6}$ involves 32 formula units ${ }^{6}$ and has a wave vector $\mathrm{q}=(0.25,0.25,0.5) 2 \pi / \mathrm{a}$, while in $\mathrm{NdB}_{6}$ there is a simple doubling 7 of the chemical unit cell. We note that the reciprocal vector in the new magnetic unit cell of $\operatorname{PrB}_{6}$ is not $(0.25,0.25,0.50) 2 \pi / \mathrm{a}$, but possesses the magnitude $1 /(2 \sqrt{2}) 2 \pi / a$ in the $\langle 110>$ direction. Earlier attempts to account for the experimental data of $\mathrm{PrB}_{6}$ by folding the paramagnetic Fermi surface into the magnetic Brillouin zone were not successful. A quantitative procedure using an analytical model for the Fermi surface is required to explore this procedure.

The $\mathrm{dHvA}$ frequency and cyclotron mass in the antiferromagnetic state of $\mathrm{PrB}_{6}$ and $\mathrm{NdB}_{6}$ are highly different from 
those in the paramagnetic state. In $\mathrm{PrB}_{6}$, the a branch is the highest frequency on the antiferromagnetic Fermi surface. This signal disappears in the angular region of $12^{\circ}$ to $35^{\circ}$ from $<100>$ in the $\{100\}$ plane and $20^{\circ}$ to $75^{\circ}$ from $\left.<100\right\rangle$ in the $\{110\}$ plane. We suppose that the nearly spherical Fermi surface of the a branch possesses large diameter arms centered in the $\langle 111\rangle$ direction and at $23^{\circ}$ from $\langle 100\rangle$ in $\{100\}$ plane. The $b, b^{\prime}, b^{\prime \prime}$ branches with a center at the $\langle 111\rangle$ direction may be related to the arms. The carrier concentration of the nearly spherical a branch roughly corresponds to that of one ellipsoid in the paramagnetic state. As the carrier concentration does not change below the Neel temperature, the arms in the a branch and other Fermi surfaces may compensate the carrier concentration contributed by the remaining two ellipsoids.

In $\mathrm{NdB}_{6}$ the signal of the a branch, the highest frequency in the antiferromagnetic state, disappears in the angular region of $40^{\circ}$ to $90^{\circ}$ in the $\{110\}$ plane, and in a narrow angular range of approximately $1^{\circ}$ centered at $\langle 110\rangle$ in the $[100\}$ plane. This branch is approximately the same in area as the a branch in $\mathrm{PrB}_{6}$, but the angular ranges over which it disappears are different, suggesting that its topology is different from $\mathrm{PrB}_{6}$. Of the other branches, $\mathrm{g}$ and $\mathrm{h}$ have the strongest signals and are observed at all angles, suggesting that they arise from a nearly spherical closed sheet. The other orbits exist in finite angular ranges centered at $\langle 111\rangle,\langle 110\rangle$ or $\langle 100\rangle$, except for the b orbit which does not appear near a symmetry direction. This pattern of orbits is not similar to that observed in $\mathrm{PrB}_{6}$, suggesting that the Fermi surface topology in the antiferromagnetic state of the two metals is not the same. This conclusion is consistent with the very different magnetic structure and unit cell in the two materials.

In the antiferromagnetic states of $\mathrm{PrB}_{6}$ and $\mathrm{NdB}_{6}$, the masses do not follow simple scaling laws. In both materials, the masses are rather heavy, as shown by the a orbits which have masses of $2.52 \mathrm{~m}_{0}$, and $2.00 \mathrm{~m}_{0}$ respectively. While these masses are large compared to the non- $\mathrm{LaB}_{6}$, they are not large compared to typical transition metal compounds where magnetism is observed. Therefore it is possible that the antiferromagnetic modifications to the Fermi surface band structure of these metals involve mostly d-sp hybridization. 


\section{CONCLUSION}

We have measured the electrical resistivity, Hall coefficient, magnetoresistance and the de Haas - van Alphen effect of $\mathrm{PrB}_{6}$ and $\mathrm{NdB}_{6}$ to study their Fermi surface properties. The experimental results are as follows:

1) The Hall coefficient is temperature independent in the range of $0.7 \mathrm{~K}$ to room temperature. The number of conduction electrons is approximately one per unit cell. This is consistent with the Fermi surface model of $\mathrm{LaB}_{6}$, where a set of three electron ellipsoids centered at $\mathrm{X}$ and connected by small necks in the $\mathrm{X}-\mathrm{X}$ direction contain one electron per unit cell. Even below the Neel temperature, the carrier concentration as measured by the Hall effect does not change.

2) The magnetoresistance shows that both substances are uncompensated metals where the carriers are electrons. Open orbits are found at many angles in the $[100\}$ and $[110\}$ planes, implying a multiply connected Fermi surface in $\mathrm{PrB}_{6}$ and $\mathrm{NdB}_{6}$. The $\left.<100\right\rangle$ direction is a singular field direction in $\mathrm{NdB}_{6}$ but not in $\mathrm{PrB}_{6}$. 3) In $\mathrm{PrB}_{6}$ we have observed the antiferromagnetic Fermi surface reflecting the smaller magnetic Brillouin zone induced by the magnetic structure, as well as the paramagnetic Fermi surface which can be seen due to magnetic breakdown through the small antiferromagnetic gaps. The paramagnetic Fermi surface consists of three ellipsoids which are the same as those of $\mathrm{LaB}_{6}$. The necks interconnecting between ellipsoids are, however, larger than those of $\mathrm{LaB}_{6}$, which removes the singular field direction at $\langle 100\rangle$. The main antiferromagnetic Fermi surface is a nearly spherical Fermi surface with arms along the $<111>$ directions.

4) In $\mathrm{NdB}_{6}$, only the antiferromagnetic Fermi surface has been observed, which is highly different from those of $\mathrm{PrB}_{6}$ and $\mathrm{LaB}_{6}$. The main Fermi surface is a nearly spherical sheet which is not observed over the whole angular range.

5) The cyclotron mass of the paramagnetic main Fermi surface (ellipsoid) in $\operatorname{PrB}_{6}, 1.95 \mathrm{~m}_{0}$, is three times larger than that of $\mathrm{LaB}_{6}$, . $0.61-0.64 \mathrm{~m}_{\mathrm{o}}$.

6) The cyclotron masses of the antiferromagnetic main, nearly spherical Fermi surfaces in $\mathrm{PrB}_{6}$ and $\mathrm{NdB}_{6}$ are $2.52 \mathrm{~m}_{\mathrm{o}}$ and $2.00 \mathrm{~m}_{\mathrm{o}}$, respectively. Although these masses are slightly heavier than those in 
the paramagnetic state, they are similar to those observed in other magnetic d electron metals.

\section{Acknowledgements}

We are grateful to B. I. Min, M. R. Norman, D. D. Koelling, Y. Ishizawa and A. Hasegawa for their helpful discussions. This work was supported by the U.S. Department of Energy, BES-Materials Sciences under contract W-31-ENG-38 and also by the Grand-In-Aid for the Scientific Research from the Ministry of Education, Science and Culture in Japan. 


\section{REFERENCES}

1. T. Kasuya, Theory of Heavy Fermion and Valence Fluctuations, Ed. by T. Kasuya and T. Saso, (Springer-Verlag, Berlin Heidelberg, 1985), p. 2.

2. D. D. Koelling, B. D. Dunlap, and G. W. Crabtree, Phys. Rev. B31, 4966 (1985).

3. G. W. Crabtree, J. Mag. Mag. Mat. 52, 169 (1985).

4. T. Komatsubara, N. Sato, S.Kunii, I. Oguro, Y. Furukawa, Y. Onuki, and T. Kasuya, J. Mag., Mag. Mat. 31-34, 368 (1983).

5. S. Horn, F. Steglich, M. Loewenhaupt, H. Scheuer, W. Felsch, and K. Winzer, Z. Phys. B42, 125 (1981).

6. C. M. McCarthy, C. W. Thompson, R. J. Graves, H. W. White,Z. Fisk and H. R. Ott, Solid State Commun. 36, 861 (1980).

7. C. M. McCarthy, C. W. Thompson, J. Phys. Chem. Solids 41,1319 (1980).

8. G. W. Crabtree, H. Aoki, W. Joss, and F. Hulliger, Proceedings of the $5^{\text {th }}$ International Conference on Valence Fluctuations, 5-9 January, 1987, Bangalore, India.

9. W. R. Johanson, G.W. Crabtree, A. S. Edelstein and O. D. McMasters, J. Mag. Mag: Mat. 31-34, 377 (1983); W. R. Johanson, G.W. Crabtree, A. S. Edelstein, and O. D. McMasters, Phys. Rev. Lett. 46, 504 (1981); G. W. Crabtree, W. R. Johanson, A. S. Edelstein, and O. D. McMasters, in Valence Fluctuations in Solids, eds. L. M. Falicov, W. Hanke, and M. B. Maple (North . Holland, Amsterdam,1981) p.93.

10. P. H. P. Reinders, M. Springford, P. T. Coleridge, R. Boulet, and D. Ravot, Phys. Rev. Lett. 57, 1631 (1986).

11. L Taillefer, R. Newbury, G. G. Lonzarich, Z. Fisk, J. L. Smith, J. Mag. Mag. Mat. 63-64, 372 (1987).

12. A. J. Arko, G. W. Crabtree, D. Karim, F. M. Mueller, L. R. Windmiller, J. B. Ketterson and Z. Fisk, Phys. Rev. B13, 5240 (1976).

13. A. J. Arko, G. Crabtree, J. B. Ketterson, F. M. Mueller, P. F. Walch, L. Windmiller, Z Fisk. R. Hoyt, A. Mota, R. Viswanathan, D. E. Ellis, A. J. Freeman, and J. Roth, Int. J. Quant. Chem. Symp.2 569 (1975):

14. Y. Ishizawa, T. Tanaka, E. Bannai and S. Kawai, J. Phys. Soc. Japan 42, 112 (1977).

15...Y. Ishizawa, H. Nozaki, T. Tanaka and T. Nakajima, J. Phys. Soc. 
Japan 48, 1439 (1980).

16. P. F. Walch, D. E. Ellis, and F. M. Mueller, Phys. Rev. B15, 1859 (1977).

17. A. Hasegawa and A. Yanase, J. Phys. F2, 1245 (1977).

18. A. P. J. van Deursen, Z. Fisk and A. R. de Vrooman, Solid State Commun. 44, 609 (1982).

19. A. P. J. van Deursen, R. E. Pols, A. R. de Vrooman and Z. Fisk, J. Less-Common Metals, 111, 331 (1985).

20. Y. Onuki, M. Nishihara, M. Sato and T. Komatsubara, J. Magn. and Magn. Mater. 52, 317 (1985).

21. N. Sato, A. Sumiyama, S. Kunii, H. Nagano and T. Kasuya, J. Phys. Soc. Japan 54, 1923 (1985).

22. N. Sato, S. Kunii, I. Oguro, T. Komatsubara and T. Kasuya, J. Phys. Soc. Japan 53,3967 (1984).

23. T. Tanaka, E. Bannai, S.Kawai and T. Yamane, J. Crystal Growth 30 , 193 (1975).

24. Y. Ishizawa et al., Researching Report of National Institute for Research in Inorganic Materials, No. 17 (1978) (in Japanese).

25. N. Sato, Thesis (Tohoku University, 1983).

26. S. Tanigawa, S. Terakado, Y. Iwase, R. Suzuki, T. Komatsubara and Y. Onuki, J. Magn. and Magn. Mater., 52, (1985) 313. 


\section{FIGURE CAPTIONS}

Fig 1: Cross section of the $\mathrm{LaB}_{6}$ Fermi surface in the $\Gamma X M$ plane.Lower case Greek letters $\alpha, \gamma, \varepsilon$ and $v$ refer to particular orbits on the surface.

Fig 2: Temperature dependence of the electrical resistivity in $\mathrm{RB}_{6}$.

Fig 3: Temperature dependence of the Hall coefficient in $\mathbf{R B}_{6}$.

Fig 4: Angular dependence of the transverse magnetoresistance of (a) $\mathrm{LaB}_{6},\left(\right.$ b) $\mathrm{CeB}_{6}$, (c) $\mathrm{PrB}_{6}$ and (d) $\mathrm{NdB}_{6}$ in the $(100)$ plane. The data of $\mathrm{LaB}_{6}$ was cited from ref. 24 .

Fig 5: Field dependence of the transverse magnetoresistance of (a) $\mathrm{LaB}_{6}$, (b) $\mathrm{PrB}_{6}$ and (d) $\mathrm{NdB}_{6}$ at typical field directions in the $\{100\}$ plane. The data of $\mathrm{LaB}_{6}$ was cited from ref. 24 .

Fig 6: Angular dependence of the transverse magnetoresistance of (a) $\mathrm{LaB}_{6},(\mathrm{~b}) \mathrm{PrB}_{6}$ and (c) $\mathrm{NdB}_{6}$ in the [110] plane.

The data of $\mathrm{LaB}_{6}$ was cited from ref. 24.

Fig 7: Field dependence of the transverse magnetoresistance of (a) $\mathrm{LaB}_{6}$, (b) $\mathrm{PrB}_{6}$ and (c) $\mathrm{NdB}_{6}$ at typical directions in the $\{110\}$ plane. The data of $\mathrm{LaB}_{6}$ was cited from ref. 24 .

Fig 8: Angular dependence of the dHvA frequencies in (a) $\mathrm{LaB}_{6}$, (b) $\mathrm{PrB}_{6}$ and (c) $\mathrm{NdB}_{6}$. The data of $\mathrm{LaB}_{6}$ was cited from refs. 14 and 15.

Fig 9: Trajectories of the open orbits in $\mathrm{LaB}_{6}$. The trajectories are labelled by 
the field directions shown in the figure, and the arrows show the open orbit directions.

Fig 10: Comparison of masses of $\mathrm{LaB}_{6}$ and of the paramagnetic Fermi surface of $\operatorname{PrB}_{6}$. 


\section{TABLE CAPTIONSI}

Table 1: Field directions at which open orbits are observed in $\mathrm{LaB}_{6}, \mathrm{PrB}_{6}$ and $\mathrm{NaB}_{6}$. The data of $\mathrm{LaB}_{6}$ was cited from ref. 24.

Table 2: dHvA frequencies and cyclotron masses in (a) $\mathrm{LaB}_{6}$ (b) $\mathrm{PrB}_{6}$ and (c) $\mathrm{NdB}_{6}$ in the field along the $<100>$ direction. Data by Ishizawa et al., Arko et al and van Deursen et al. are due to refs, 14 and 15, 12 and 13, and 18 and 19, respectively.

Table 3: Angular ranges where the $\alpha$ oscillation is not observed in $\mathrm{LaB}_{6}$ and PrB 6 The dats of $\mathrm{LaB}_{6}$ was cited from ref. 14. 
Figure 2

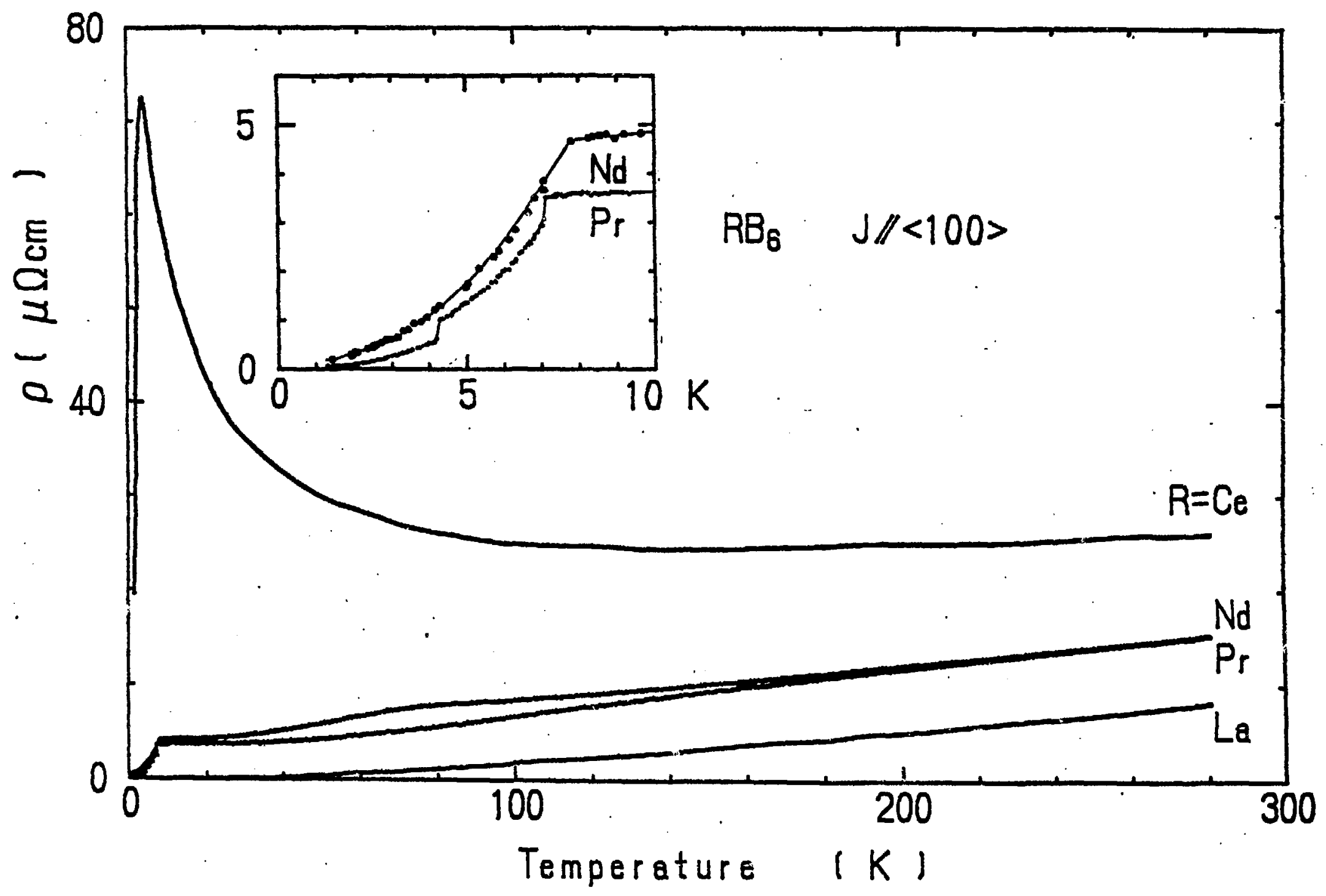




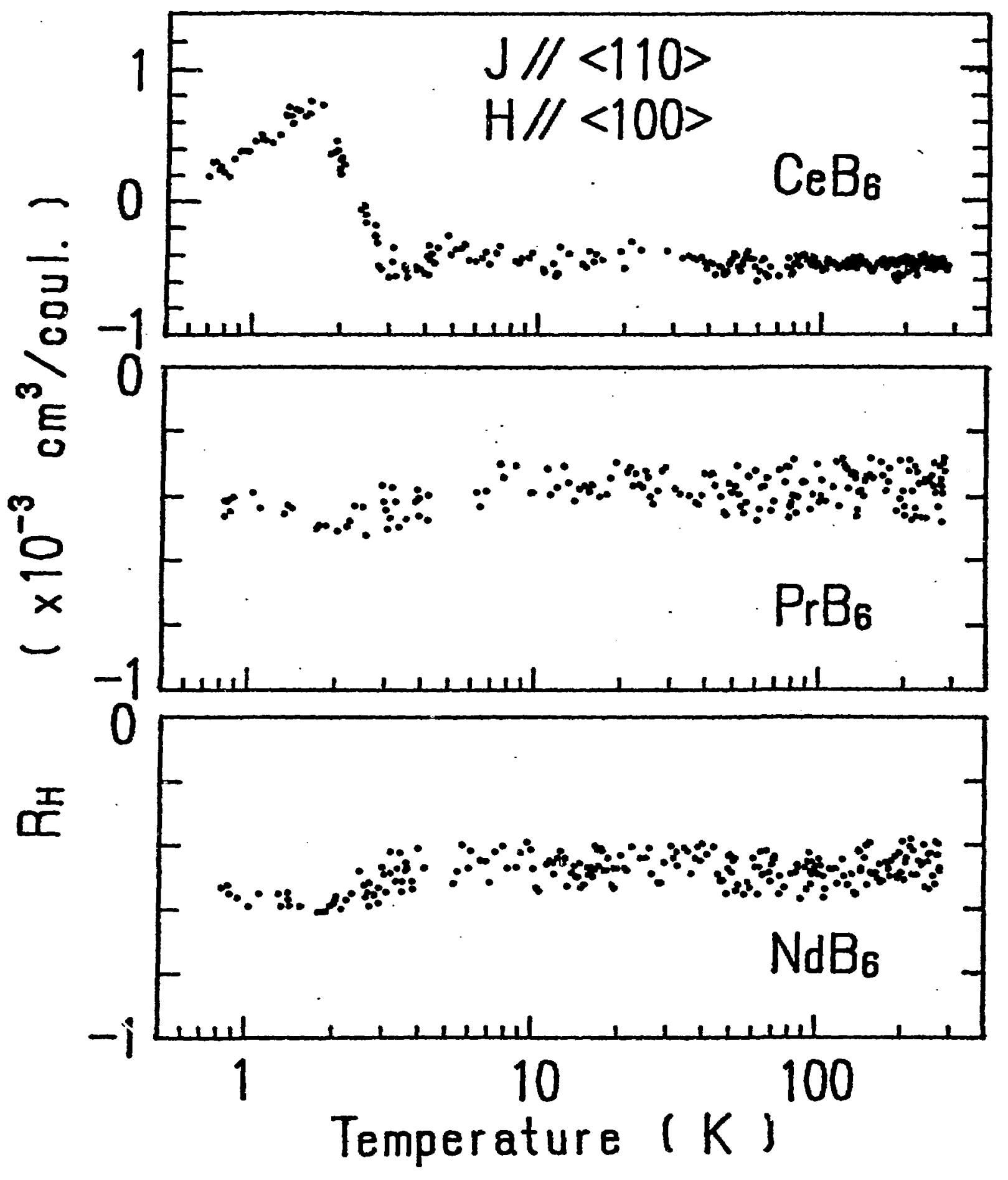




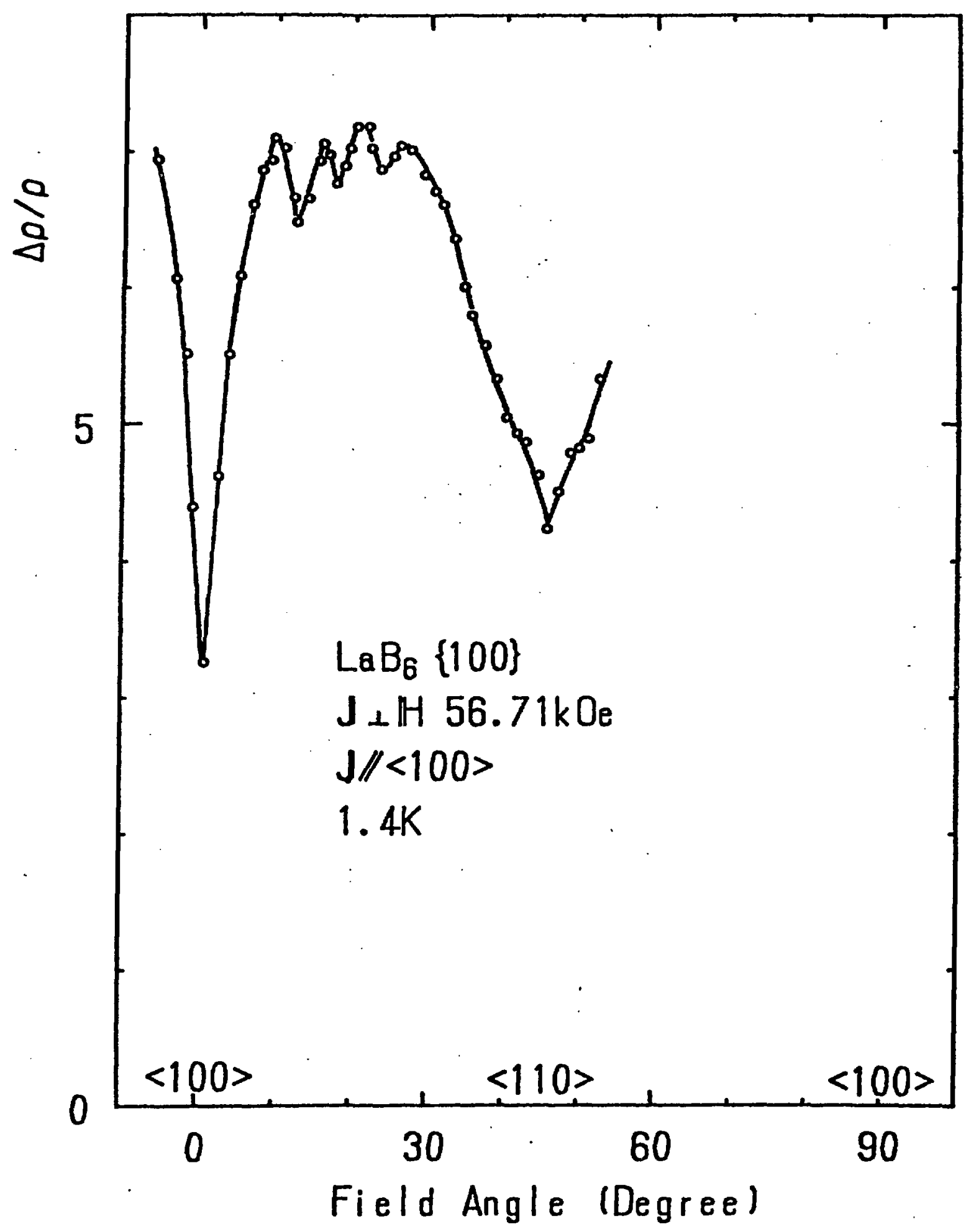




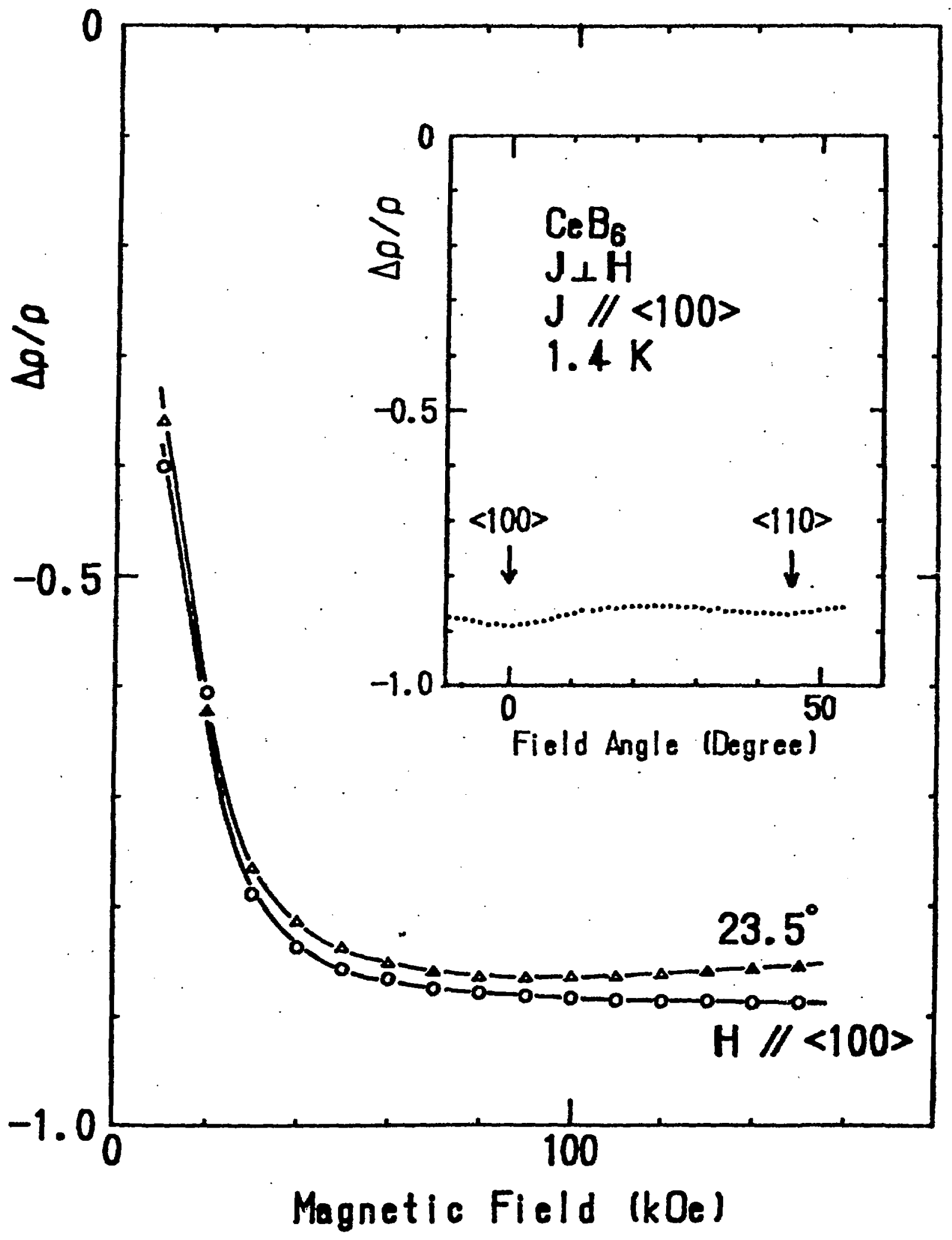




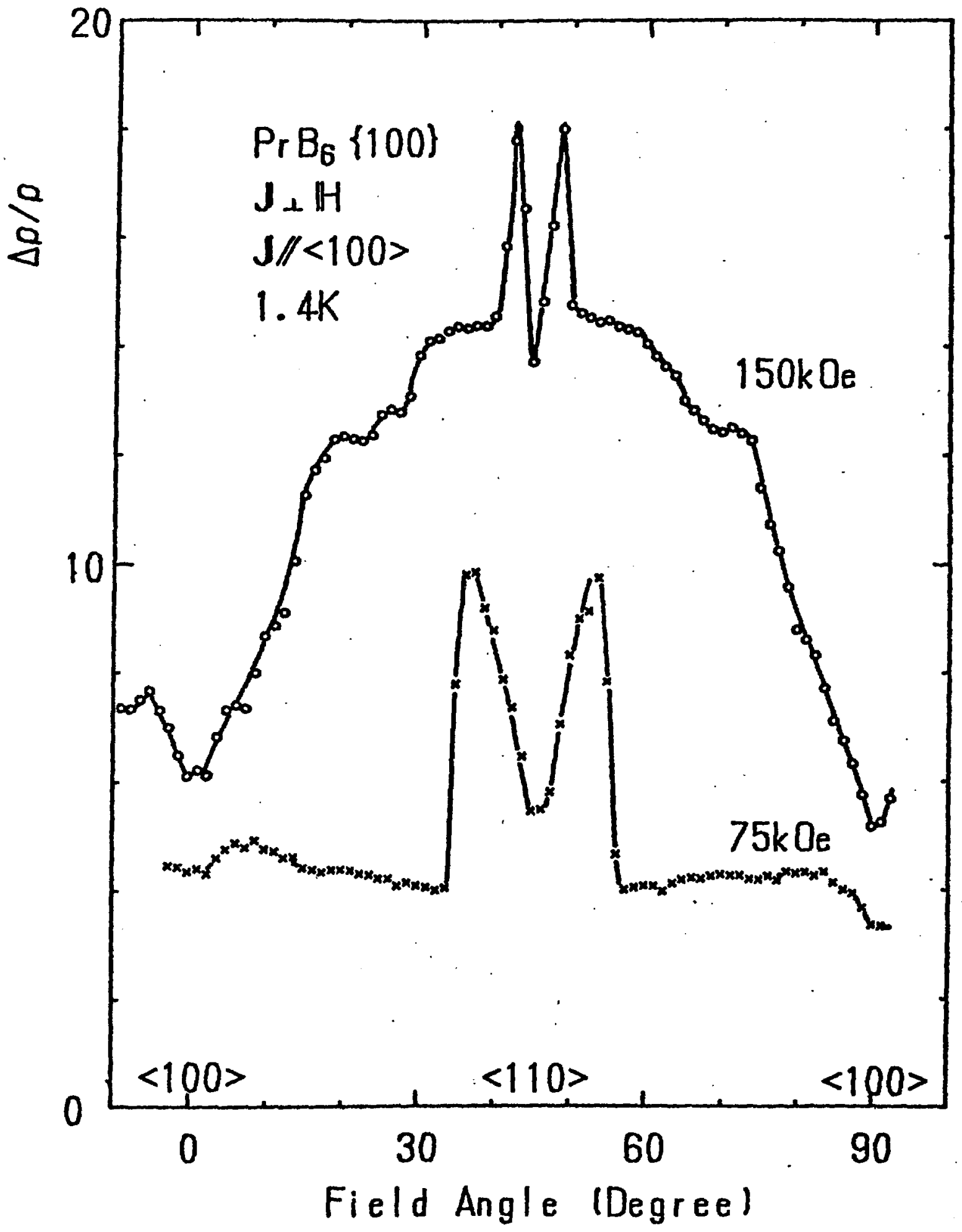

Figure $4 c$ 


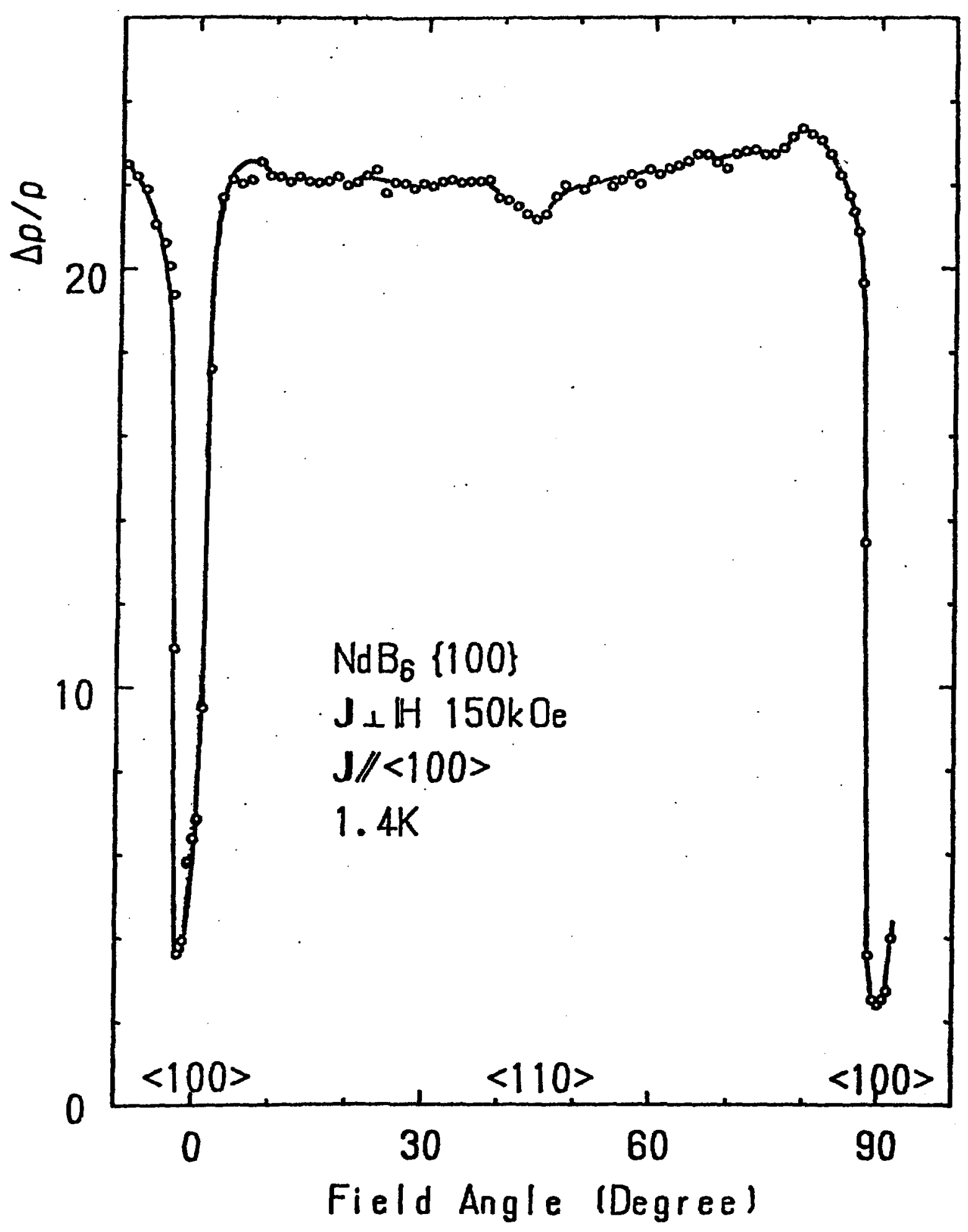




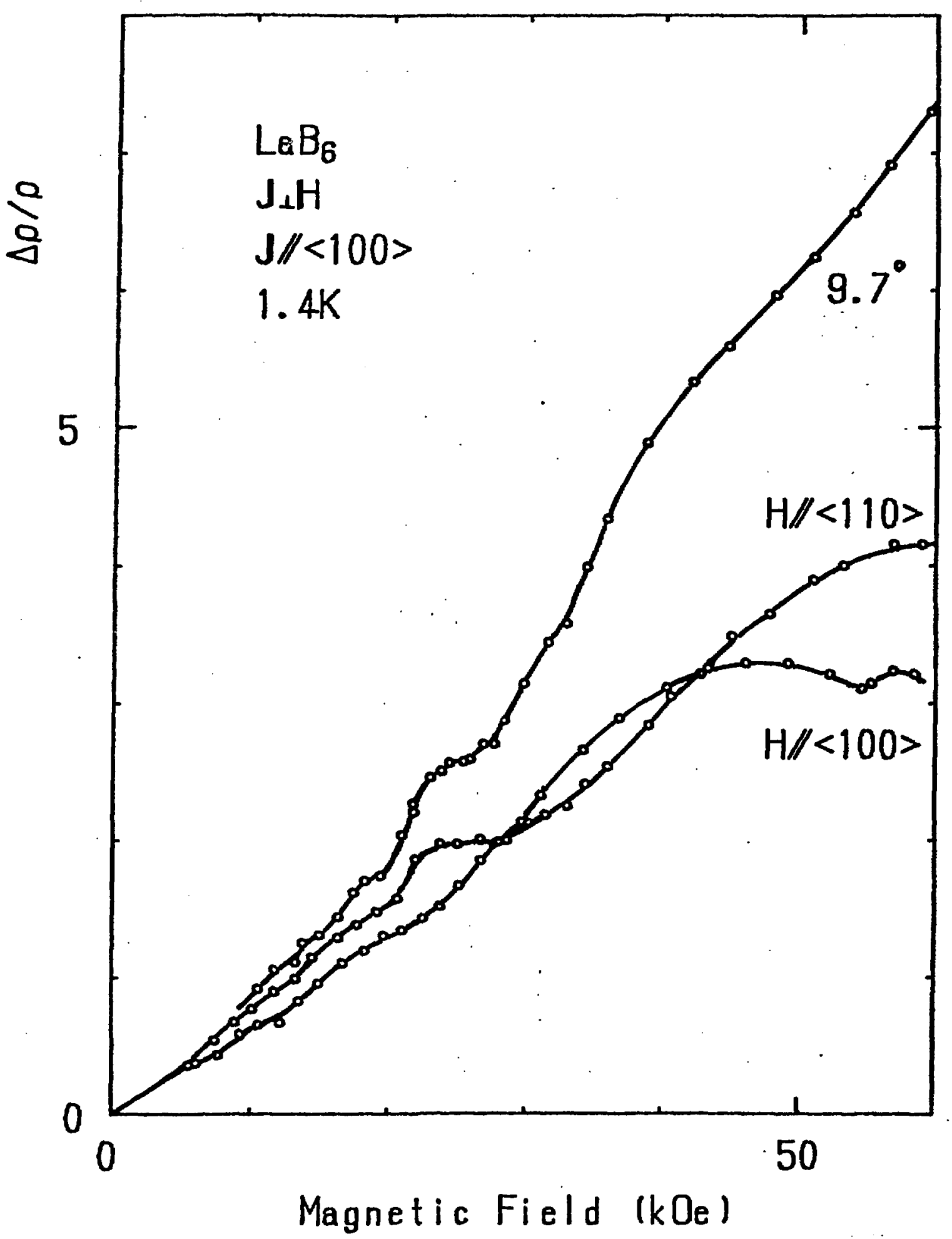

Figure $5 a$ 


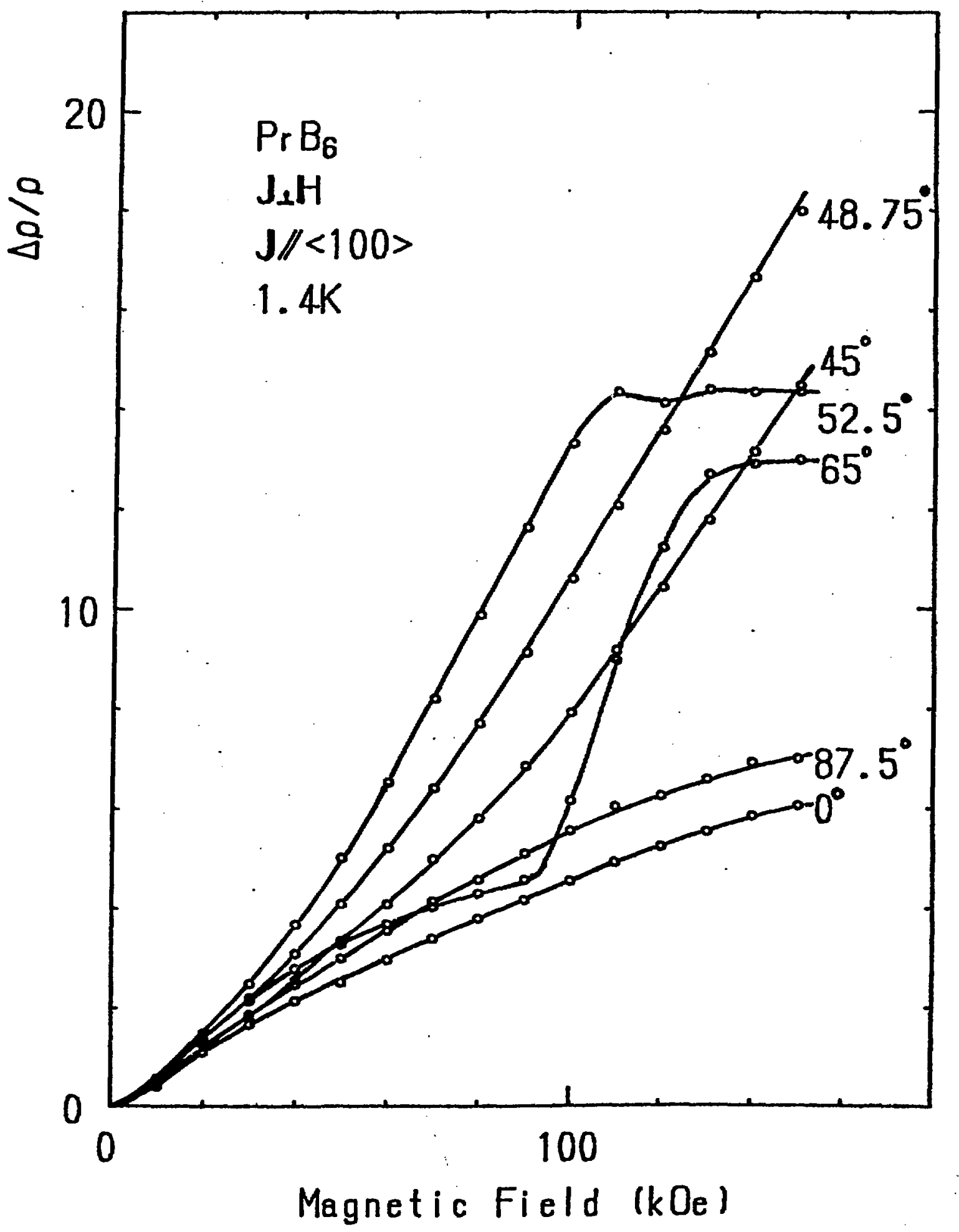

Figure 5b 


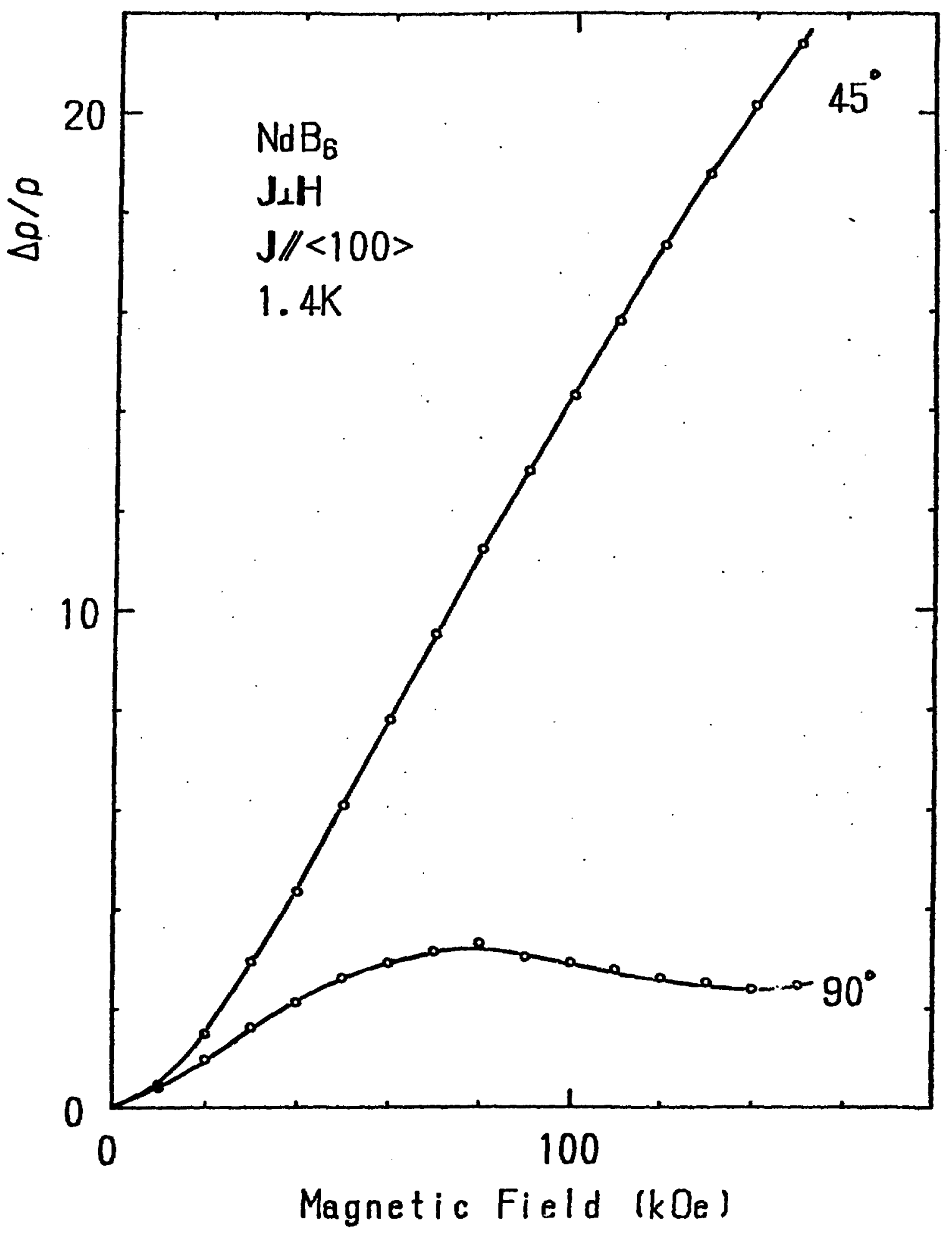

Figure 5c 


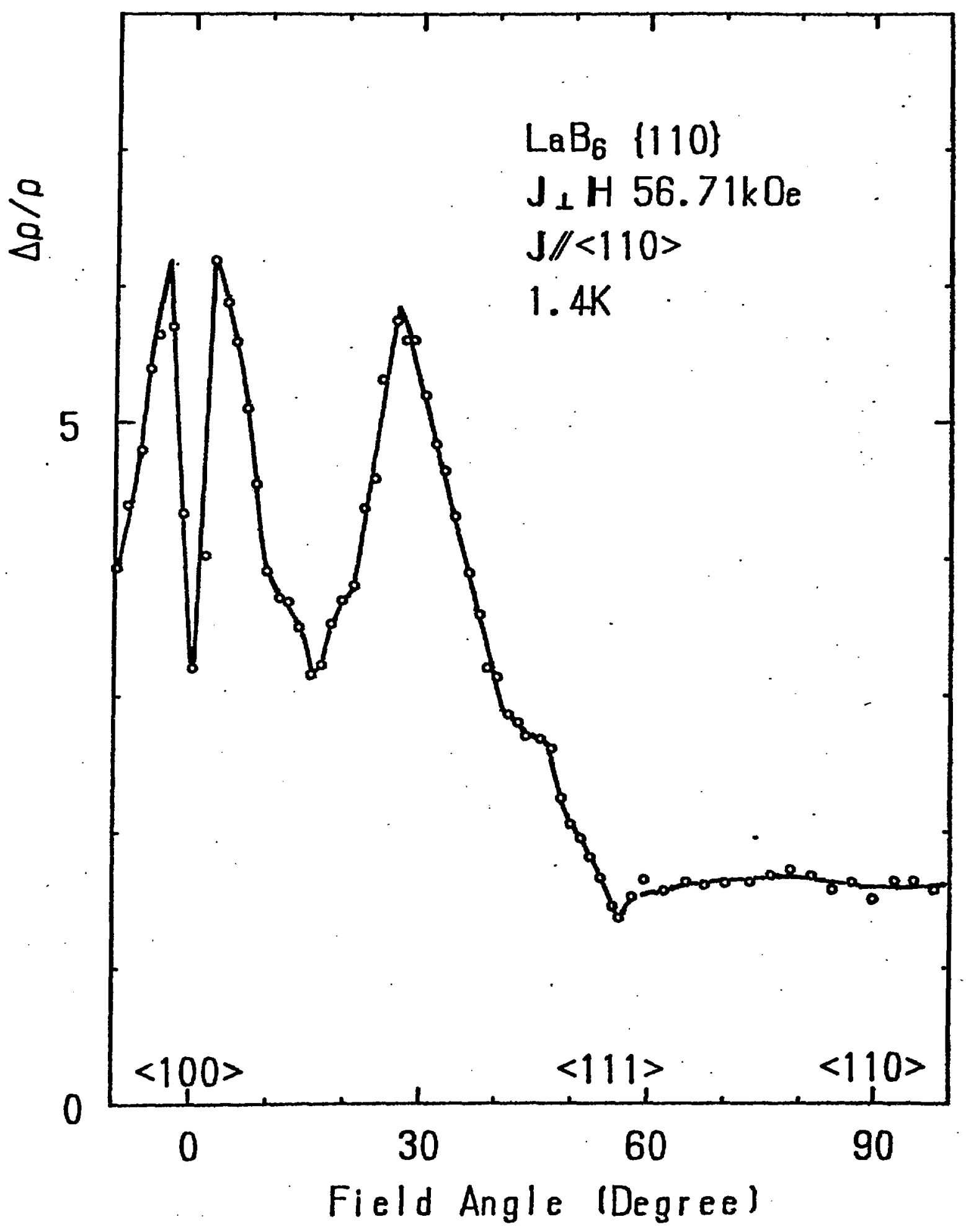

Figure 6a 


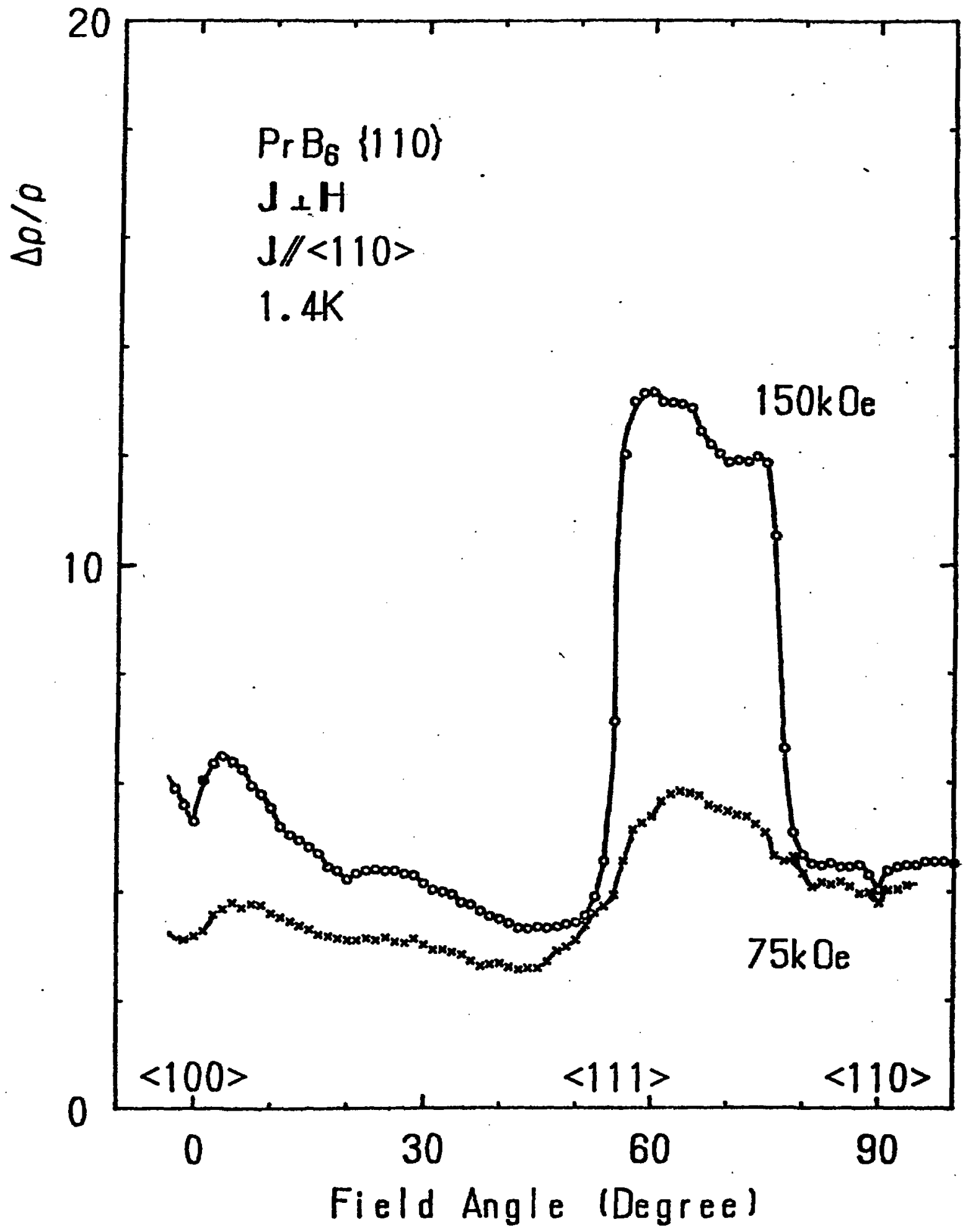




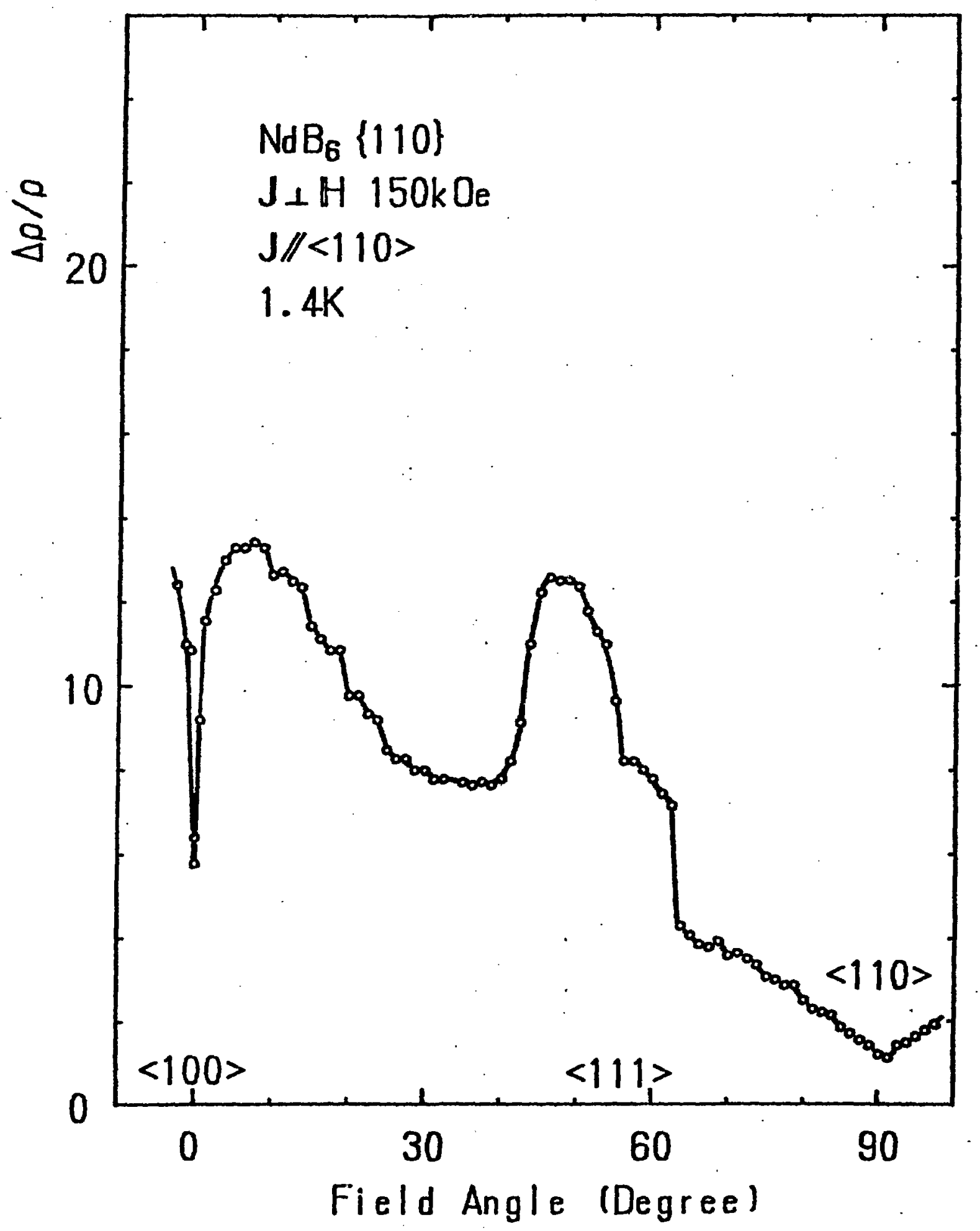

Figure $6 \mathrm{c}$ 


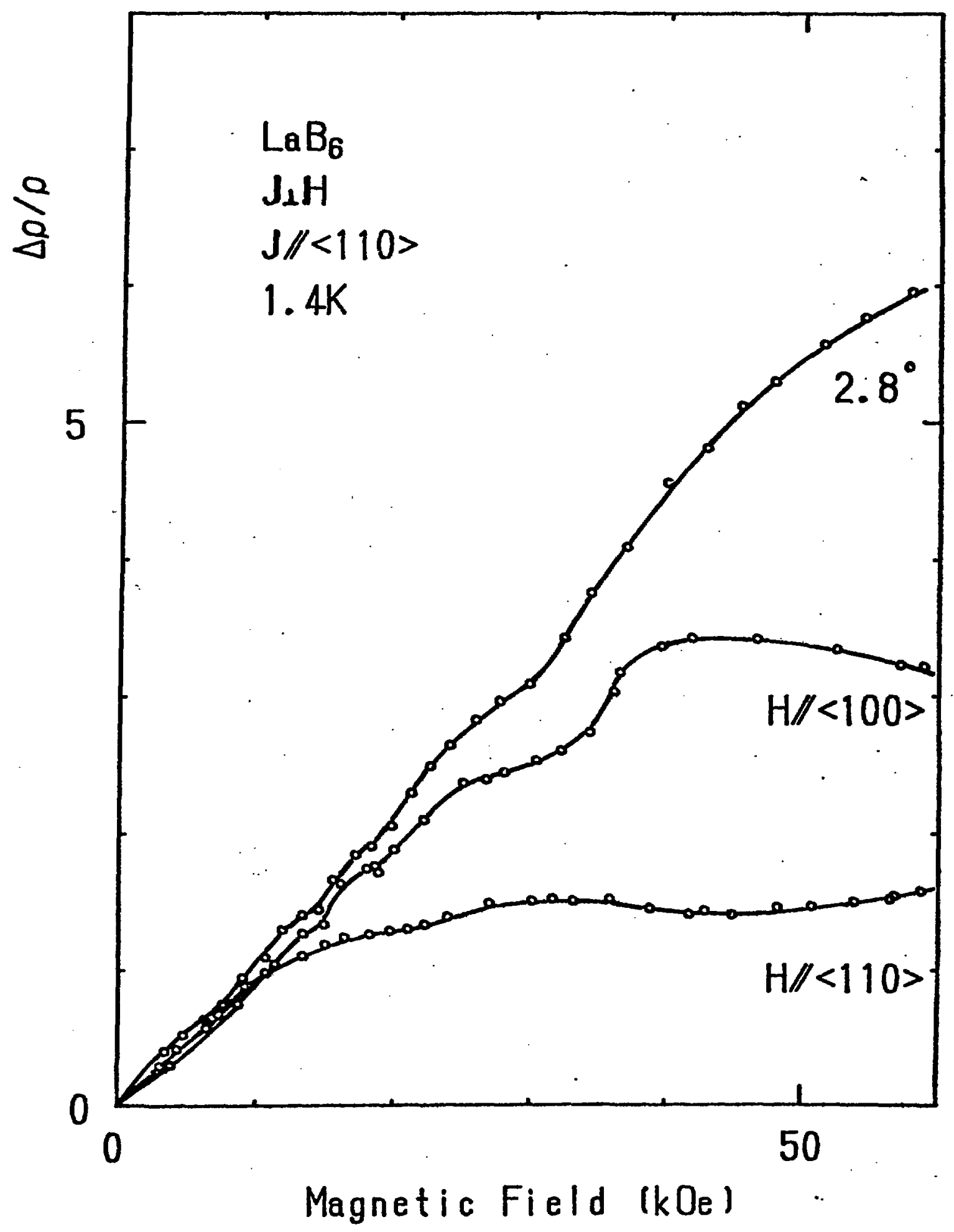




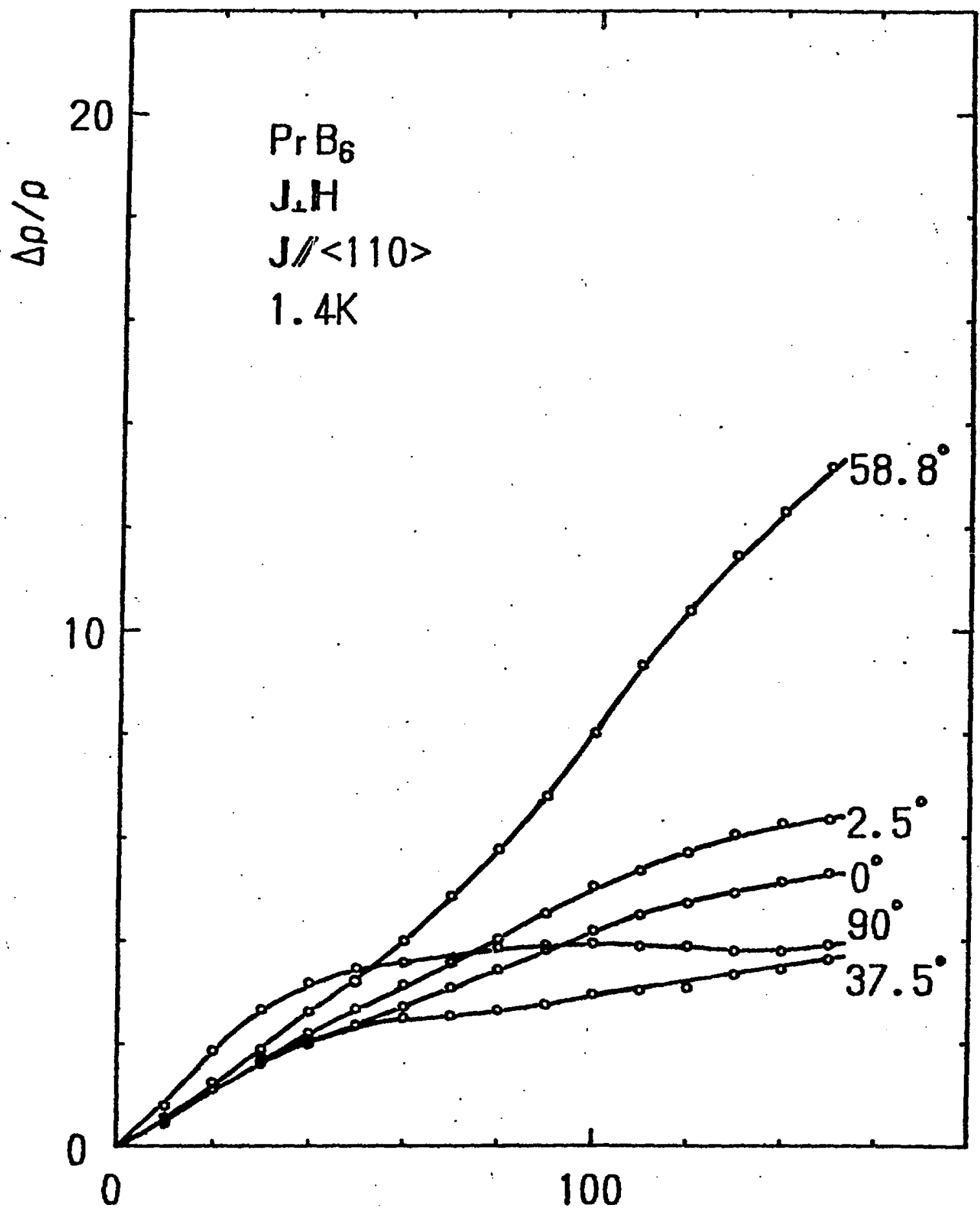

Magnetic Field ( $k \mathrm{Oe})$ 


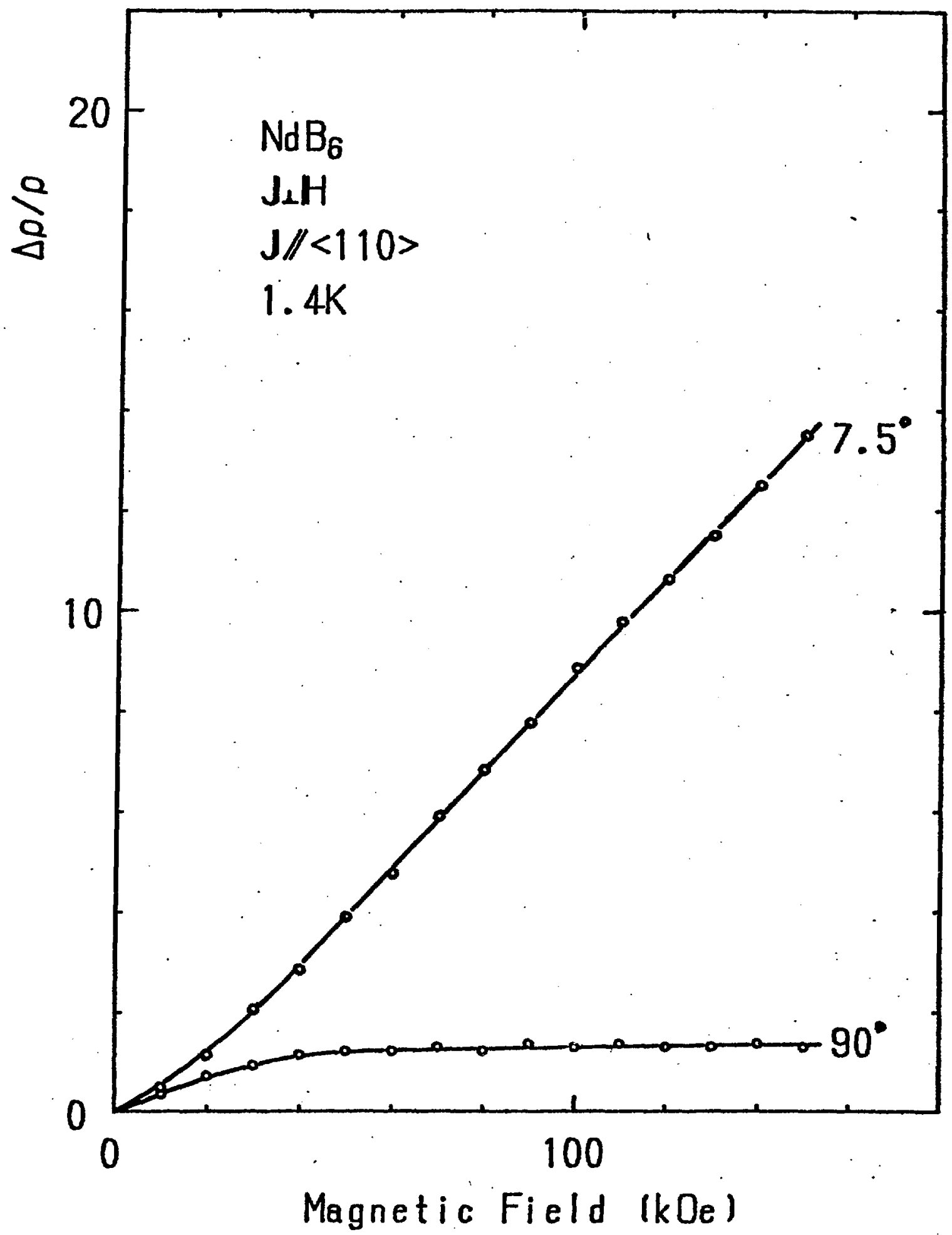

Figure $7 \mathrm{c}$ 


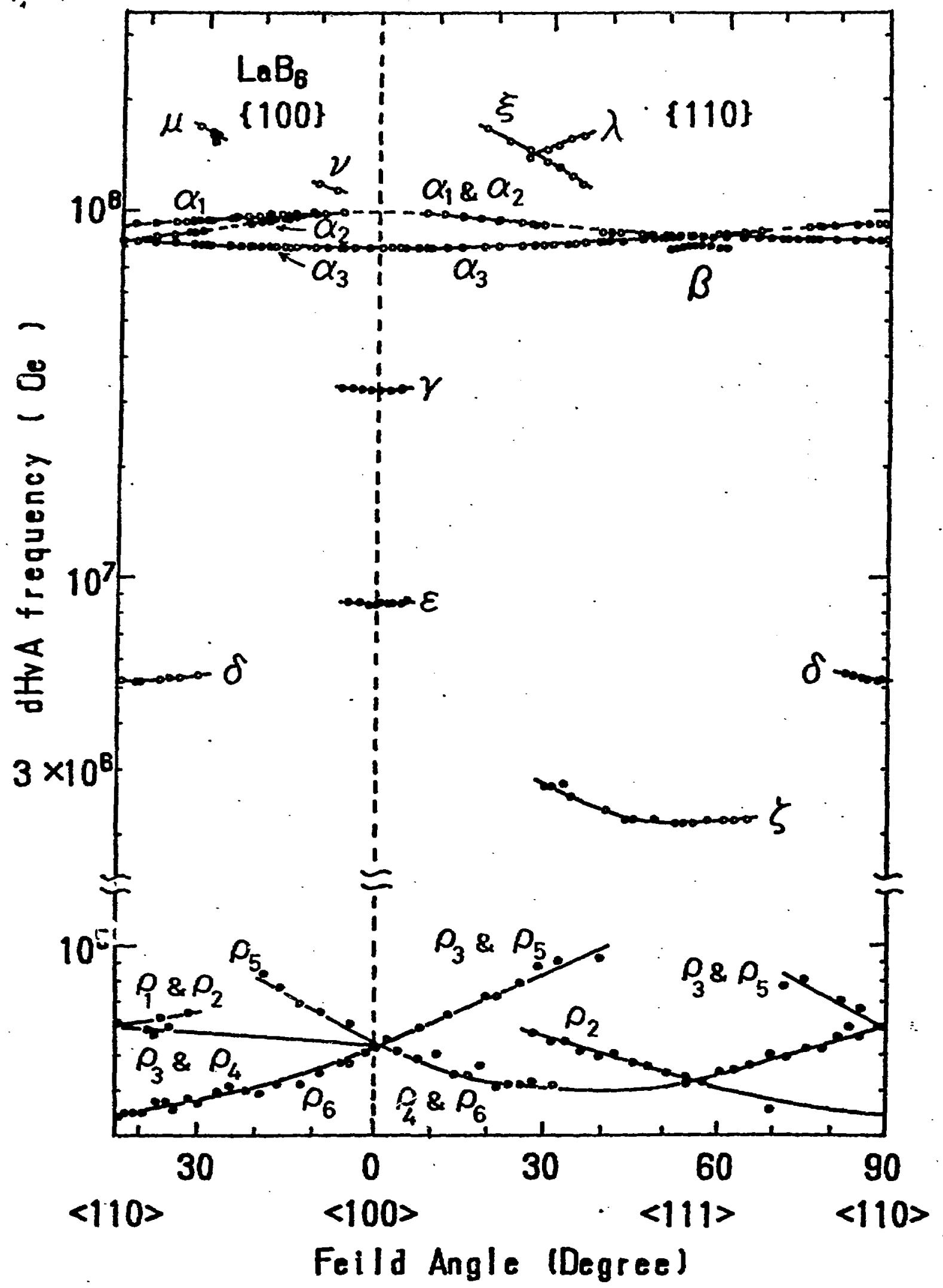

Figure $8 a$ 


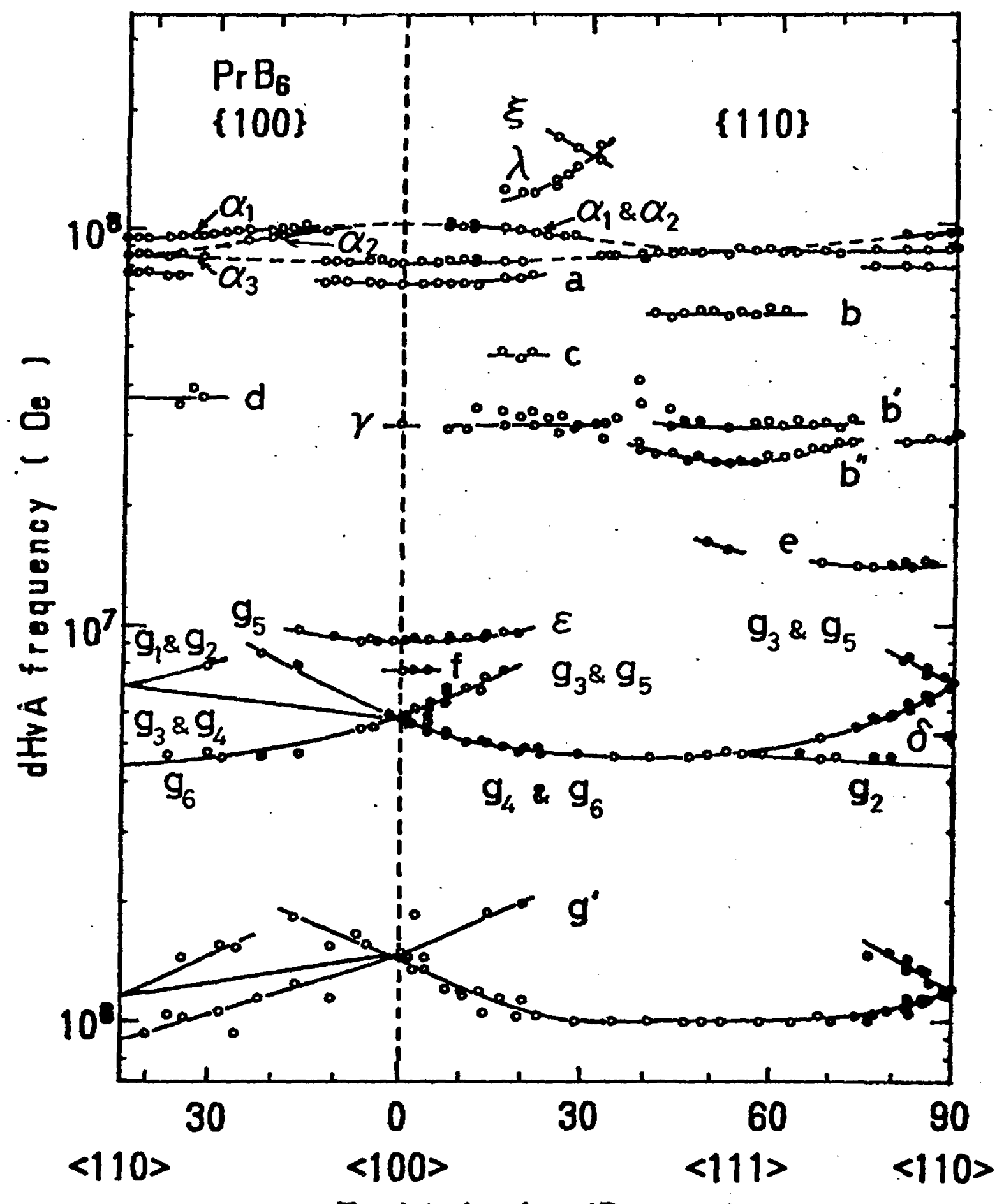

Feild Angle (Degree) 


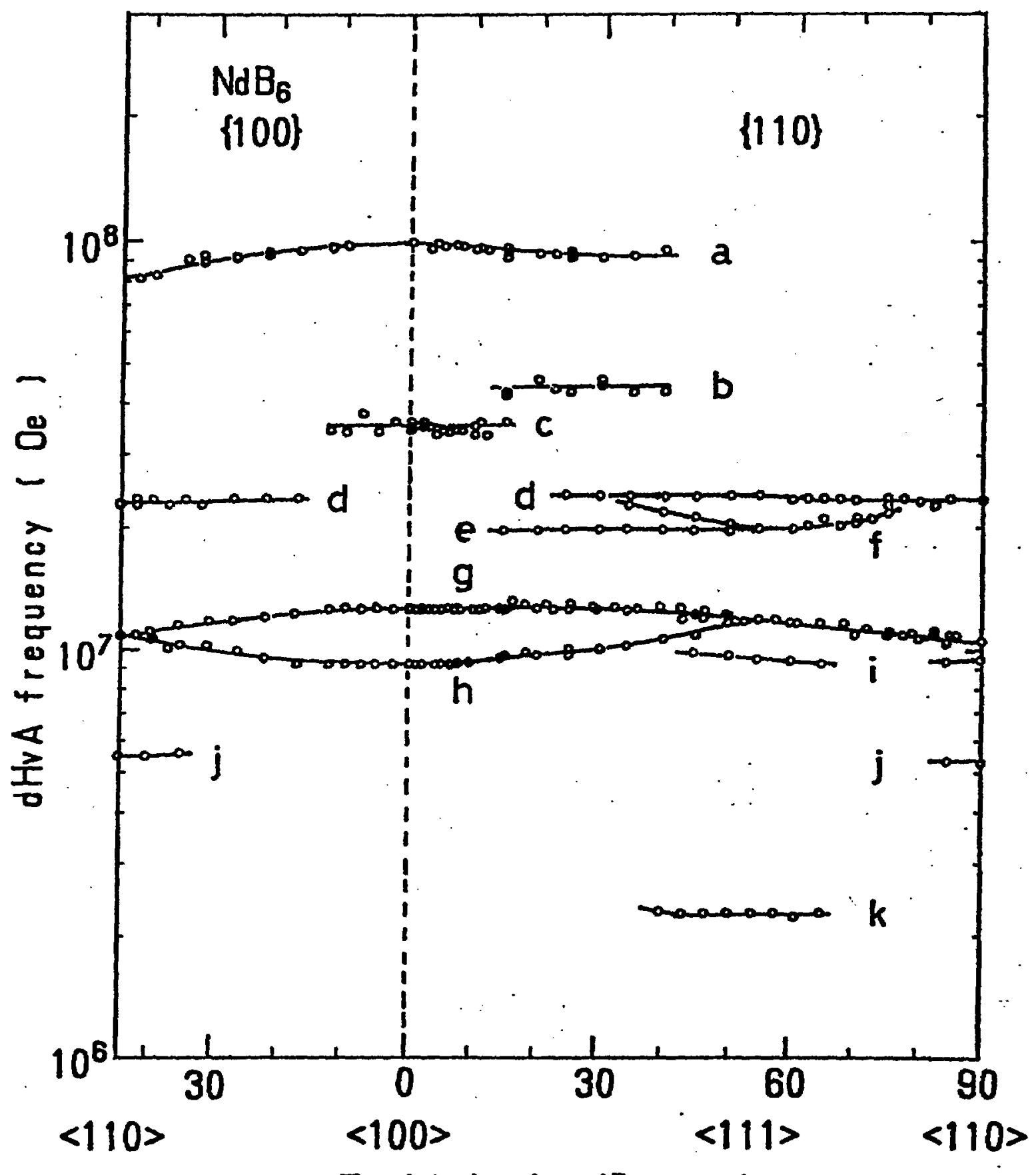

Field Angle (Degree) 
(a)

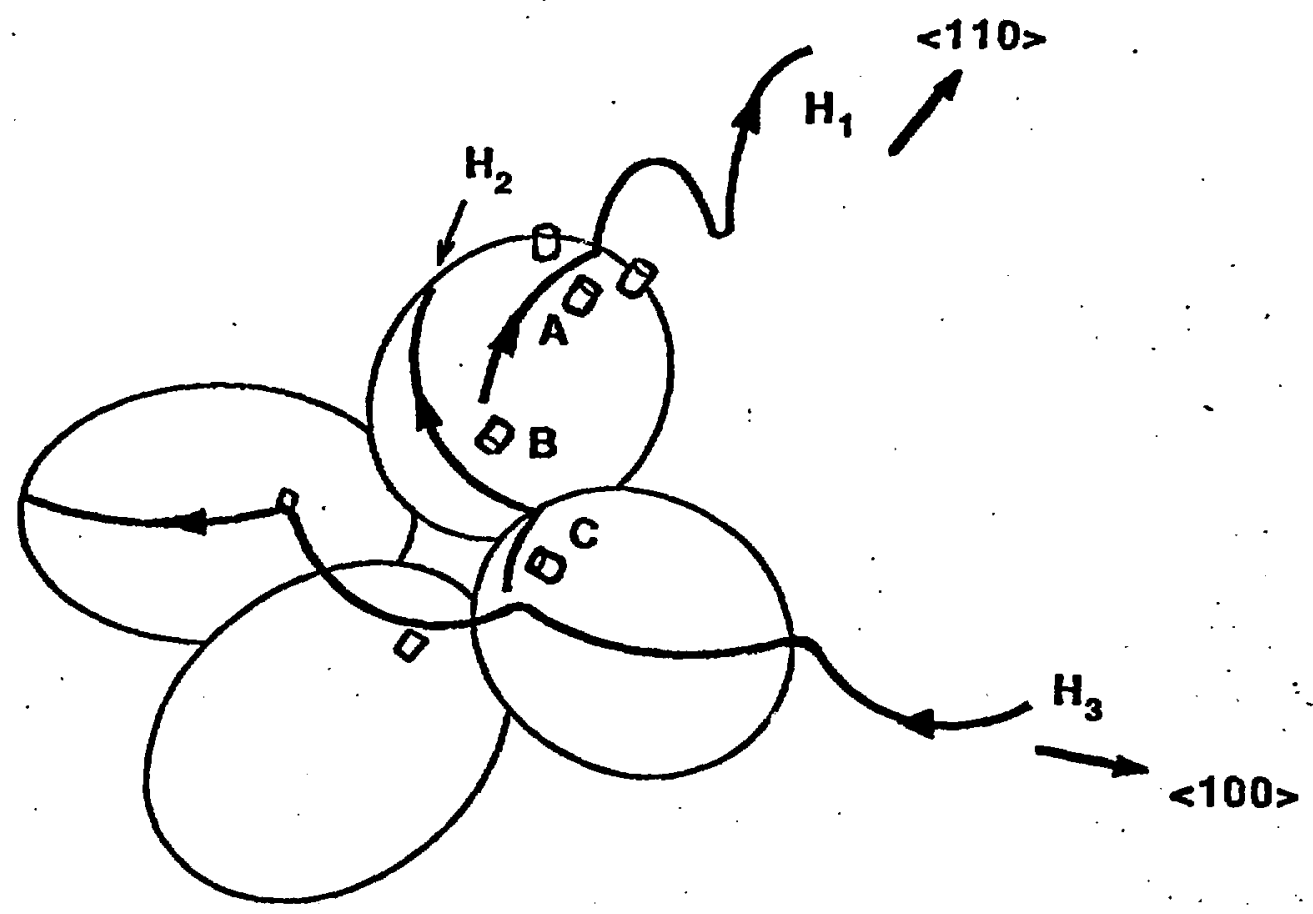

(b)
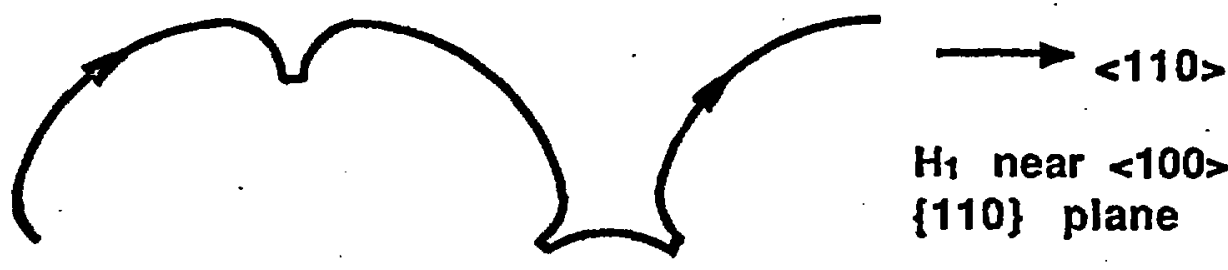

$H_{1}$ near $\left.<100\right\rangle$ in \{110\} plane

(c)

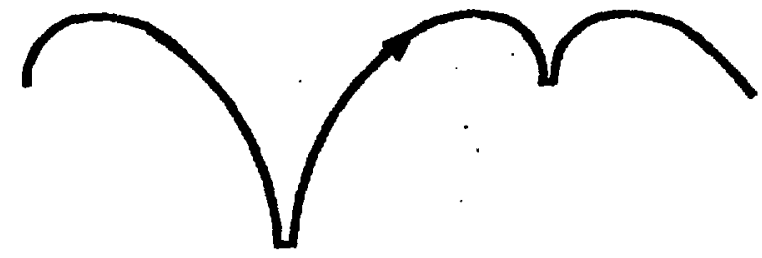

$$
\begin{aligned}
& \longrightarrow<110> \\
& 20^{\circ} \leq H_{2} \leq 35^{\circ} \text { from } \\
& <100>\text { in }\{110\} \\
& \text { plane }
\end{aligned}
$$

(d)

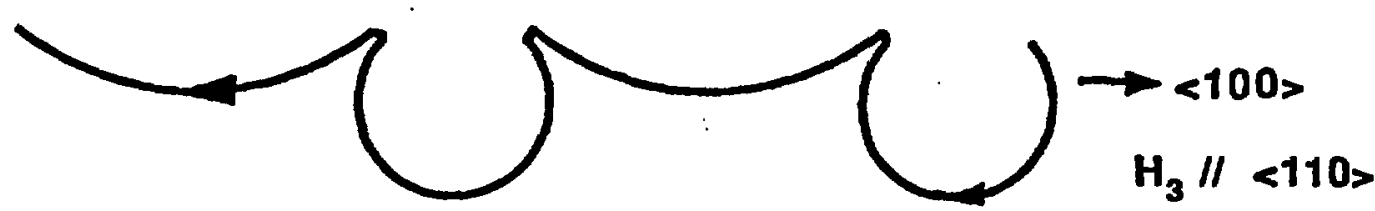




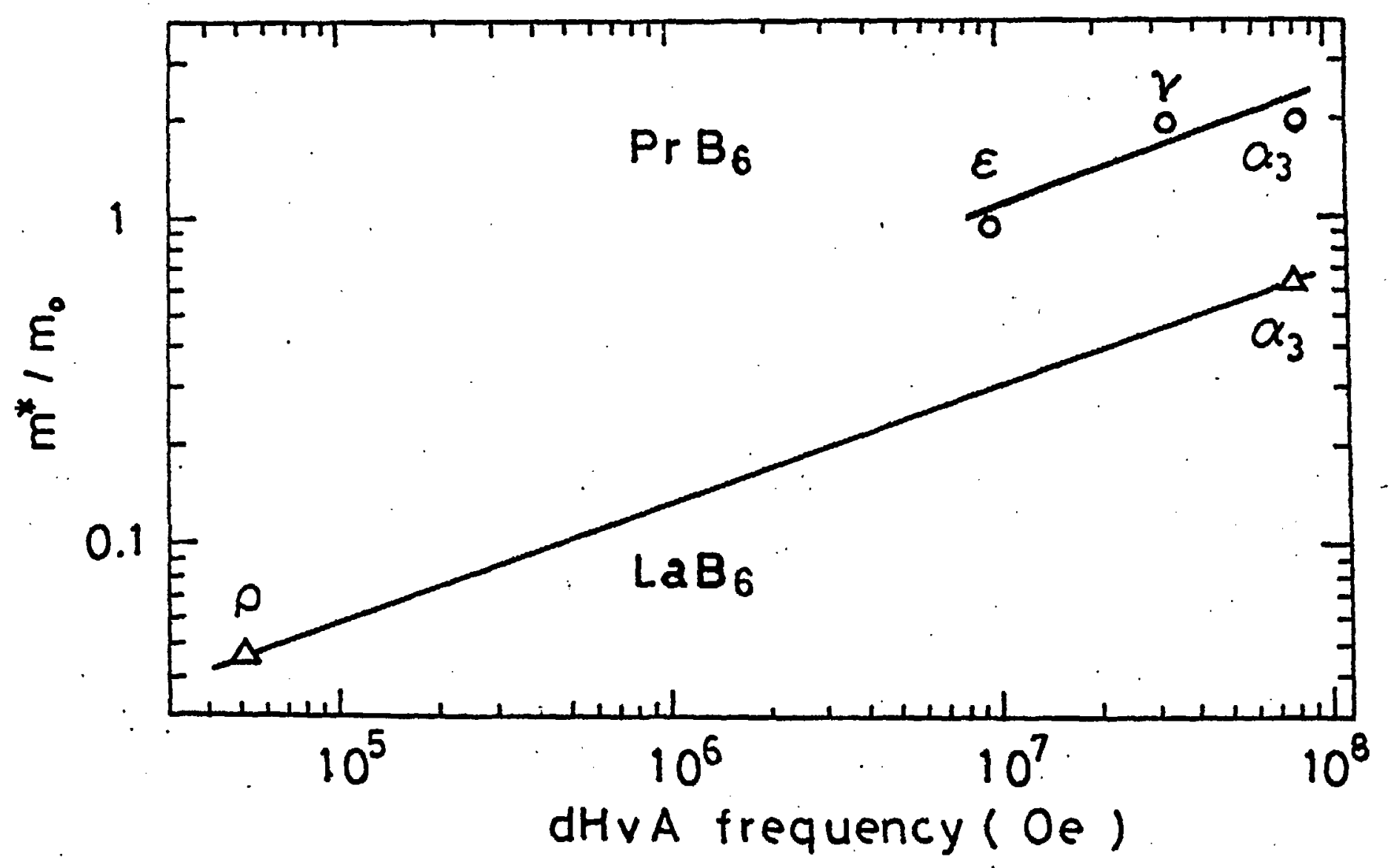

Figure 10 


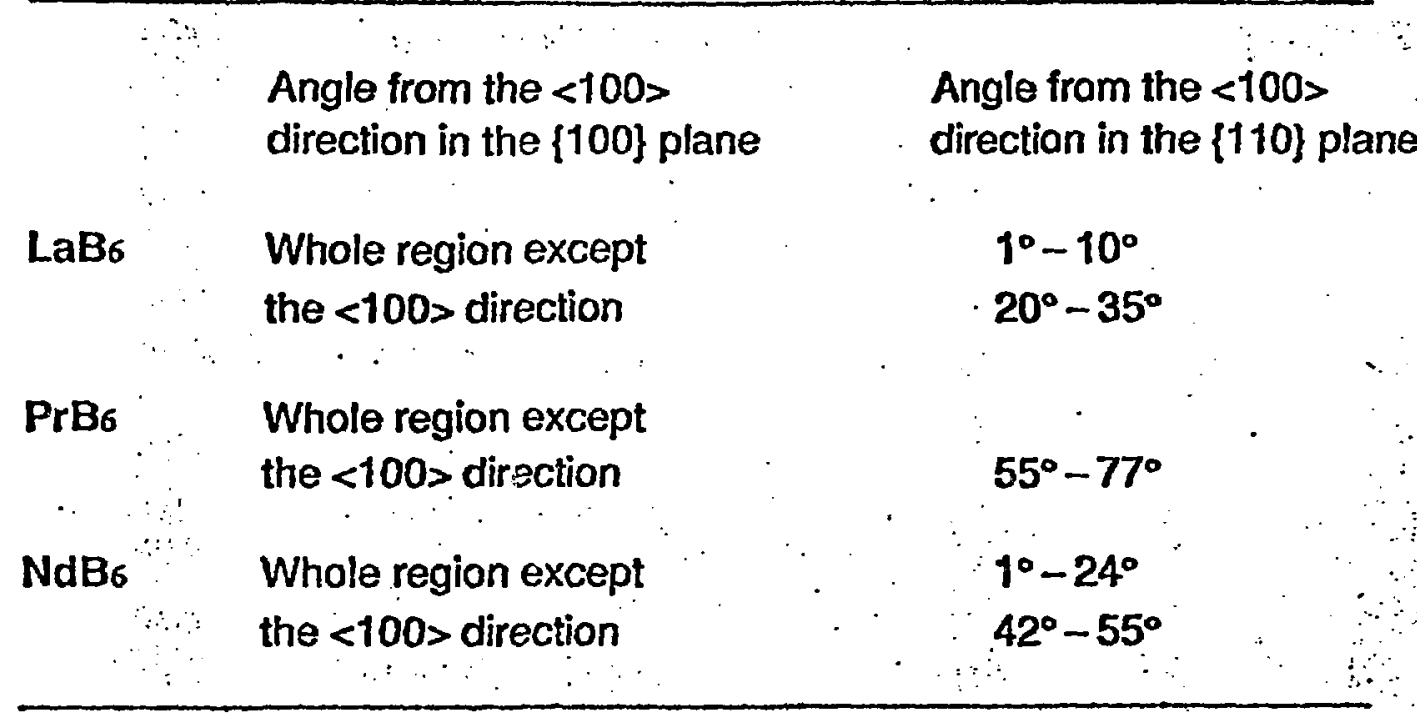

Table 1

Onubi etal. 


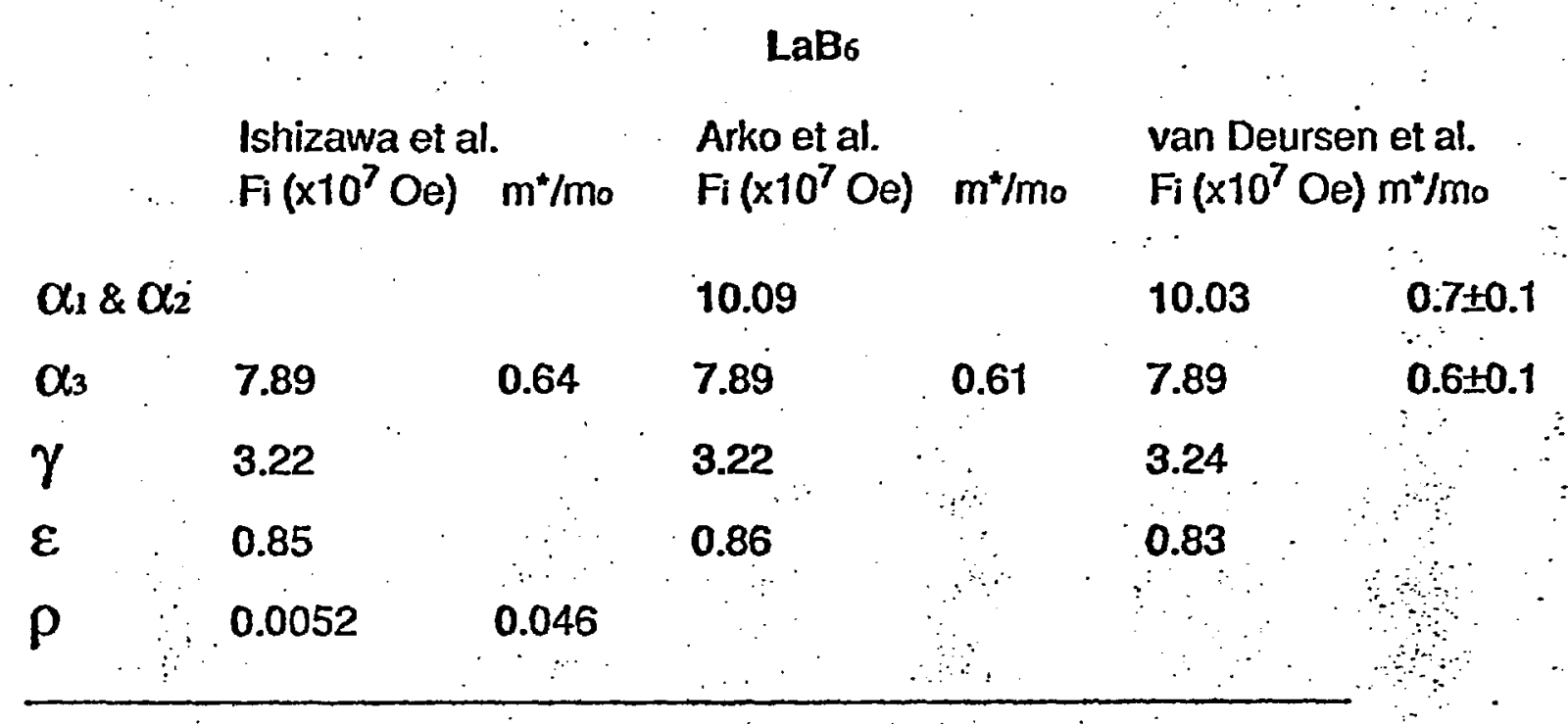

Table 2(a) Onaki etal. 


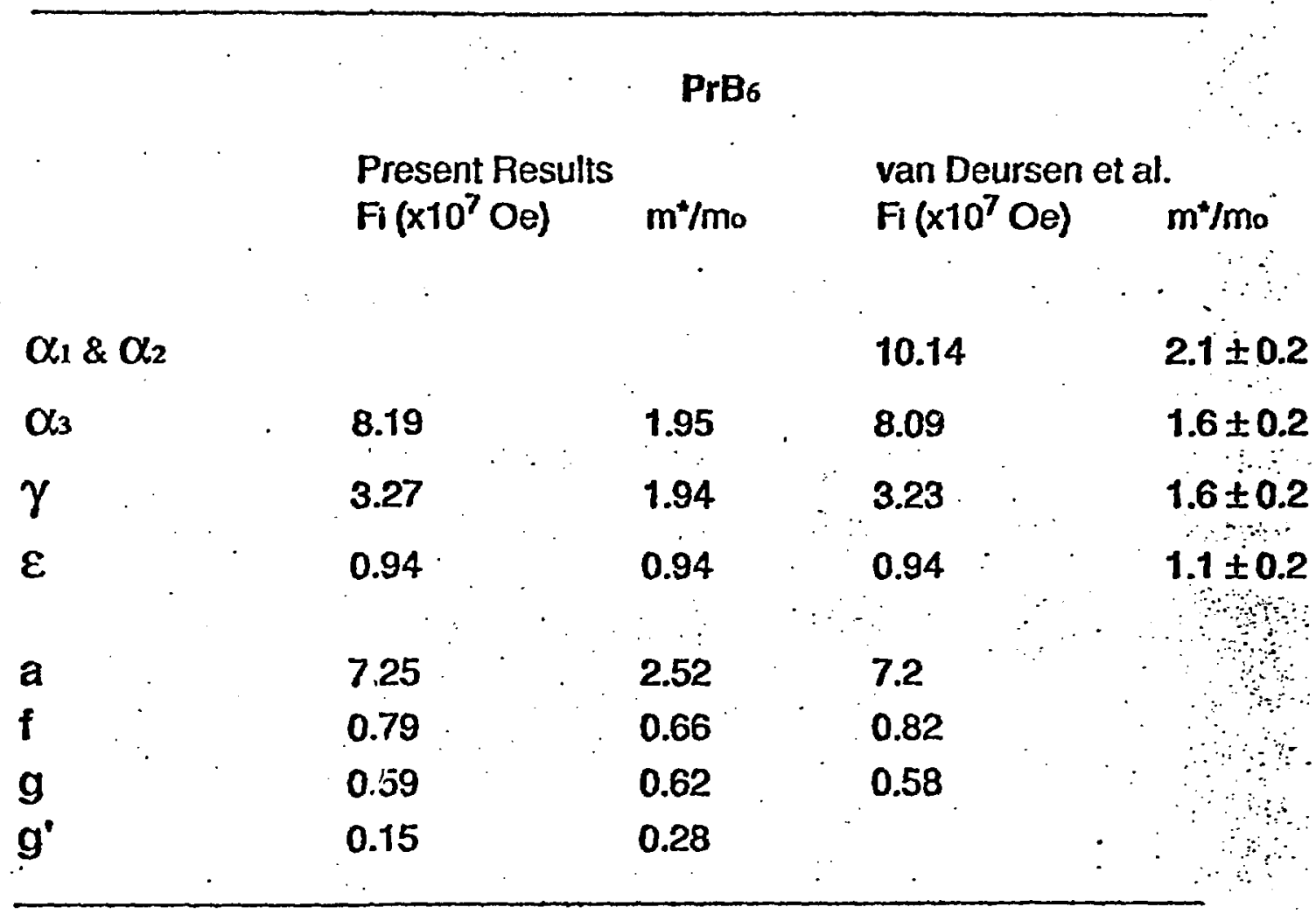

Table $2(b)$ Onuki et al. 


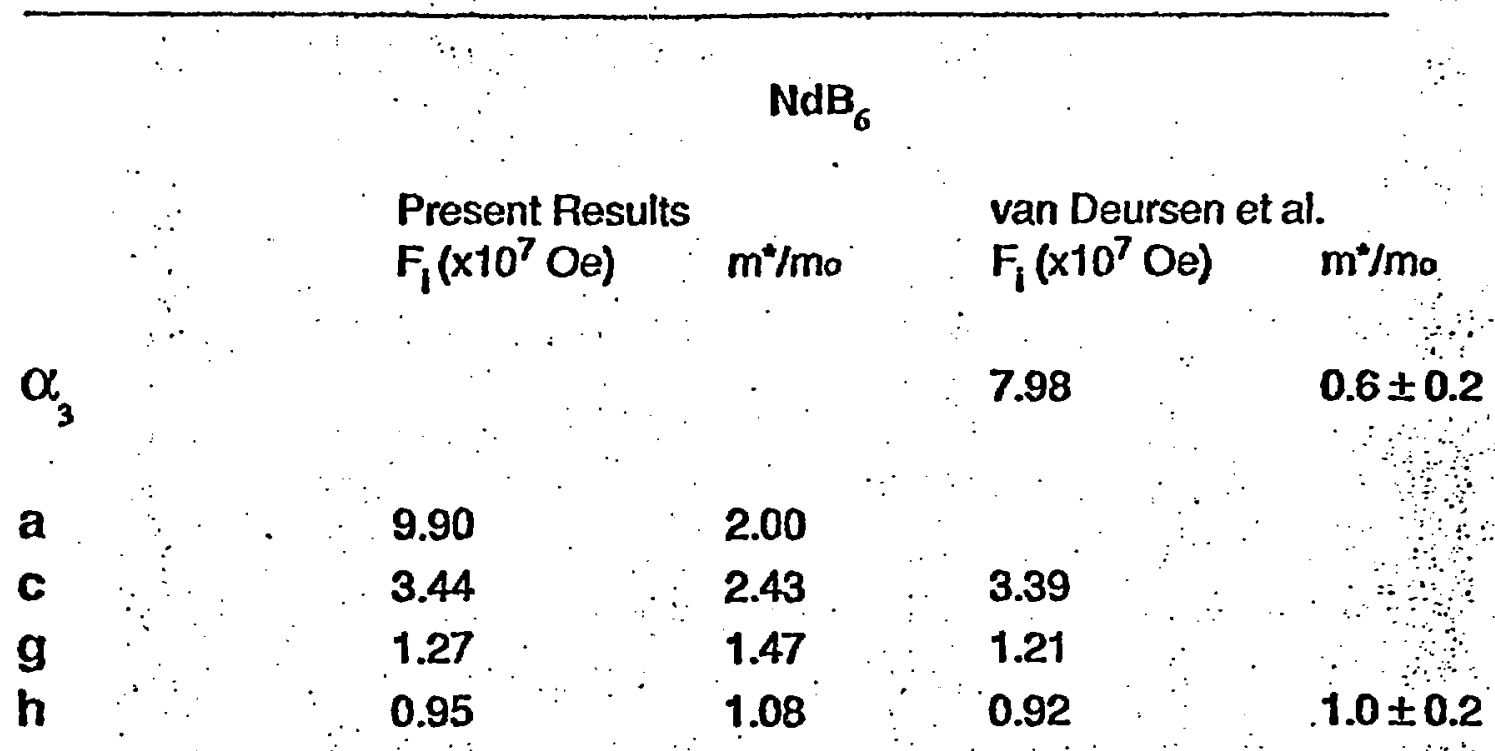

Table 2(k)

Onuki etal. 


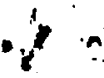

$\mathrm{LaB}_{6}$

$\mathrm{PrB}_{6}$

$\{100\}$

$\alpha_{1} \quad 0^{\circ}-9^{\circ}$

$\alpha_{2} \quad 0^{\circ}-6^{\circ} \& 23^{\circ}-30^{\circ}$

$\alpha_{3}$

\{110\}

$\left.\begin{array}{l}\alpha_{1} \\ \alpha_{2}\end{array}\right\} 0^{\circ}-9^{\circ} \& 30^{\circ}-39^{\circ}$

$\alpha_{3} \quad 68^{\circ}-75^{\circ}$ $0^{\circ}-15^{\circ}$

$0^{\circ}-12^{\circ} \& 26^{\circ}-40^{\circ}$

$15^{\circ}-30^{\circ}$

$0^{\circ}-5^{\circ} \& 30^{\circ}-40^{\circ}$

$20^{\circ}-30^{\circ} \& 60^{\circ}-80^{\circ}$

Table 3 Onuki et al. 